\title{
MAGNETICALLY CONTROLLED OUTFLOWS FROM HOT JUPITERS
}

\author{
Fred C. Adams \\ Michigan Center for Theoretical Physics, Physics Department, University of Michigan, Ann Arbor, MI 48109, USA \\ Astronomy Department, University of Michigan, Ann Arbor, MI 48109, USA \\ Kavli Institute for Theoretical Physics, University of California, Santa Barbara, CA 93106, USA \\ Received 2010 August 25; accepted 2011 January 21; published 2011 February 28
}

\begin{abstract}
Recent observations indicate that some extrasolar planets observed in transit can experience mass loss from their surfaces. Motivated by these findings, this paper considers outflows from hot Jupiters in the regime where the flow is controlled by magnetic fields. Given the mass-loss rates estimated from current observations - and from theoretical arguments-magnetic fields will dominate the flow provided that field strength near the planet is greater than $\sim 1$ G, comparable to the surface fields of the Sun and Jupiter. The problem can be separated into an inner regime, near the planet, where the outflow is launched, and an outer regime where the flow follows (primarily) stellar field lines and interacts with the stellar wind. This paper concentrates on the flow in the inner regime. For a dipole planetary field with a spatially constant background contribution, we construct a set of orthogonal coordinates that follow the field lines and determine the corresponding differential operators. Under the assumption of isothermal flow, we analytically find the conditions required for escaping material to pass smoothly through the sonic transition and then estimate the mass outflow rates. These magnetically controlled outflows differ significantly from previous spherical models. The outflow rates are somewhat smaller, typically $\dot{M} \sim 10^{9} \mathrm{~g} \mathrm{~s}^{-1}$, and the flow is launched primarily from the polar regions of the planet. In addition, if the stellar wind is strong enough, the flow could be reversed and the planet could gain mass from the star.
\end{abstract}

Key words: magnetohydrodynamics (MHD) - planets and satellites: atmospheres - planets and satellites: formation - planets and satellites: magnetic fields

\section{INTRODUCTION}

Among hundreds of extrasolar planets discovered to date, a substantial fraction orbit their stars with periods of 10 days or less. These planets are thought to have formed further out in their solar systems (e.g., Lissauer \& Stevenson 2007), and subsequently migrated inward (e.g., Papaloizou \& Terquem 2006) where they become stranded at small semimajor axes, perhaps due to disk truncation (Shu et al. 1994; Lin et al. 1996) or because the disk loses so much gas that it can no longer move planets. After planets reach these inner orbits, they are subjected to intense heating from their parental stars. This heating, which is most effective for UV photons, can drive photoevaporative flows from the planetary surfaces. In the most extreme cases, the resulting mass loss could affect both the final masses and densities of the planets. In other cases, the outflows can be observable- even if their effect on the final mass is modest - and can provide important information about planetary properties. This paper explores outflows from hot Jupiters in the regime where magnetic fields are strong enough to guide the flow and thereby determine the outflow geometry.

Mass loss from hot Jupiters has been observed in association with the transiting planet HD209458b (Vidal-Madjar et al. 2003, 2004; see also Désert et al. 2008; Sing et al. 2008; Lecavelier des Etangs et al. 2008). A recent follow-up observation using the Cosmic Origins Spectrograph on the Hubble Space Telescope implies a mass outflow rate $\dot{M} \approx 8 \times 10^{10} \mathrm{~g} \mathrm{~s}^{-1}$ from this planet (Linsky et al. 2010). In addition, signatures of atmospheric evaporation from the extrasolar planet HD189733b have recently been reported (Lecavelier des Etangs et al. 2010). More examples are expected in the term future, as well as null detections (Lecavelier des Etangs 2007). As a result, the collection of close-in extrasolar planets provides a laboratory to study the process of mass loss from planetary bodies.
Theoretical models of mass loss from extrasolar planets have been considered previously. Pioneering models of outflows from these planetary bodies have been constructed (Lammer et al. 2003; Baraffe et al. 2004, 2006) and indicate that substantial mass loss can take place. However, related studies of the effects of mass loss on the population of close-in extrasolar planets show that it is difficult to explain the observed mass distribution (Hubbard et al. 2007). In any case, a number of open questions remain. The aforementioned studies (primarily) use simple energy-limited outflow models (see also Watson et al. 1981), in conjunction with physically motivated scaling laws, and produce a range of outflow rates for given planetary masses and external UV fluxes. The next generation of theoretical calculations considered refined treatments of the chemistry, photoionization, and recombination (Yelle 2004; García Muñoz 2007), as well as the effects of tidal enhancement (Murray-Clay et al. 2009, hereafter MCM). More recently, two-dimensional models of planetary winds have been considered (Stone \& Proga 2009) and indicate that the mass loss rates can be less than those in the spherical limit. In addition to planetary outflows, alternative explanations of the observations have been put forth, where the inferred excess material is due to a confined exosphere (Trammell et al. 2011; hereafter TAL) or a mass transfer stream (Lai et al. 2010).

This theoretical investigation into planetary outflows adopts a new approach. As outlined below, the magnetic fields-from both the star and the planet-are generally strong enough to guide the flow (see also TAL). When the outflow follows the magnetic field lines, the geometry of the flow pattern is set by the field structure, but can be quite complicated. In particular, the outflows depart significantly from spherical symmetry and existing (primarily spherical) models are not applicable. In spite of this complication, the outflow problem can be reduced to one dimension by constructing a new orthogonal coordinate 
system where one coordinate follows the magnetic field lines. This approach allows for the outflow properties to be determined semi-analytically. In this context, the term "semi-analytic" refers to models where the equations are reduced to, at most, ordinary differential equations.

The problem of magnetically controlled outflows from planets can be divided into subproblems. Since the magnetic field structure of the planet guides the flow-for the regime considered here - the field is independent of the outflow and can be determined separately. Section 2 discusses the conditions required for this approach to be valid and provides an overview of the relevant scales in the problem. This work assumes that the planetary field has a dipole form. Near the planetary surface, the stellar component of the magnetic field is smaller than the planetary field and is slowly varying (spatially); as a starting approximation, we thus assume that the stellar field provides a constant background contribution. For this geometry, we construct a set of orthogonal coordinates that follow the field lines and hence the flow (Section 3). For a given field configuration, the outflow problem can be separated further into two regimes: (1) the launch of the outflow near the planetary surface; this flow depends on the heating and cooling in the vicinity of the planet, and the flow geometry is constrained by the magnetic field configuration. (2) The propagation of the outflow, away from the planet, as it enters the regime where the magnetic field structure and gravitational forces are dominated by the star. This paper focuses on the launch of the wind, in Section 4, and the propagation problem is left for future work. The observational implications of these outflows are discussed in Section 5. The paper concludes, in Section 6, with a summary of results, a discussion of their implications, and some directions for future work.

\section{OVERVIEW}

This section defines the basic scales in the problem and justifies our approach. After presenting an estimate for the outflow rate, we show that the gas is well coupled to the magnetic field and that the magnetic pressure is larger than the ram pressure of the flow by several orders of magnitude. We also discuss the background magnetic field provided by the star and the effects of the stellar wind, where both of these effects limit the sphere of influence of the planet. A background magnetic field component (from the star) is included, whereas additional contributions from currents are shown to be small.

We first make an order of magnitude estimate for the mass outflow rates from hot Jupiters using a simple scaling argument: if we assume that the outflow is limited by the rate at which the gas gains energy from the stellar UV flux, then the mechanical luminosity of the outflow $G M_{P} \dot{M} / R_{P}$ must balance the rate of energy deposition, $\eta_{\mathrm{rad}} F_{\mathrm{UV}} \pi R_{P}^{2}$, where the parameter $\eta_{\mathrm{rad}}$ includes the efficiency of energy capture and takes into account the fact that radiation can be absorbed above the planetary surface $\left(\right.$ at $R_{P}$ ). The resulting mass outflow rate $\dot{M}$ is thus given by

$$
\dot{M}=\eta_{\mathrm{rad}} \frac{\pi R_{P}^{3} F_{\mathrm{UV}}}{G M_{P}},
$$

where this expression would be exact if one could determine the correct value of $\eta_{\text {rad }}$. Next, we note the coincidence that a particle of mass $\mu$ living at the surface of a Jovian planet has potential energy $\epsilon_{\mu}$ given by

$$
\epsilon_{\mu}=\frac{G M_{P} \mu}{R_{P}} \approx 13.9 \mathrm{eV},
$$

where we have used typical values $\mu=m_{P}, M_{P}=1 M_{J}$, and $R_{P}=10^{10} \mathrm{~cm}$ to evaluate the energy. This potential energy scale is almost the same as the ionization energy for Hydrogen atoms. As a result, we can write the mass outflow rate in the form

$$
\begin{aligned}
\dot{M} & =\left(\eta_{\mathrm{rad}} \frac{\langle h \nu\rangle}{\epsilon_{\mu}}\right) \mu\left(\frac{\pi R_{P}^{2} F_{\mathrm{UV}}}{\langle h \nu\rangle}\right) \\
& \approx 1.5 \times 10^{10} \mathrm{~g} \mathrm{~s}^{-1}\left(\frac{F_{\mathrm{UV}}}{450 \mathrm{erg} \mathrm{s}^{-1} \mathrm{~cm}^{-2}}\right),
\end{aligned}
$$

where the first (dimensionless) term in brackets is close to unity and the second term in brackets represents the number of UV photons intercepted by the outflow per unit time. The UV flux is scaled to the benchmark value $F_{\mathrm{UV}}=450 \mathrm{erg} \mathrm{s}^{-1} \mathrm{~cm}^{-2}$, the flux appropriate for the quiet Sun at a distance of $a=0.05 \mathrm{AU}$ (e.g., MCM; Woods et al. 1998). The resulting numerical estimate for the outflow rate agrees with previous results (e.g., García Muñoz 2007). When the efficiency $\eta_{\text {rad }}$ is high, the outflow rate is thus determined by an approximate balance with one outgoing particle per incoming UV photon.

The above estimate ignores magnetic fields, whereas this paper considers the wind to be guided by the field. In order for the plasma to be well coupled to the magnetic field, the cyclotron frequency $\omega_{C}$ must be larger than the collision frequency $\Gamma$. The cyclotron frequency is given by $\omega_{C}=B q /(m c)$ and the collision rate is given by $\Gamma=n \sigma v$. For the parameter space of interest, we expect the magnetic field strength near the planet to be $B \sim 1-40 \mathrm{G}$ (e.g., TAL) and the collision cross section to be $\sigma \sim 2 \times 10^{-13} \mathrm{~cm}^{2}$ (see Shu 1992; Spitzer 1978; Sturrock 1994). Because the continuity equation implies that $\dot{M}=4 \pi r^{2} \rho v$, we can write the ratio of frequencies in the form

$$
\begin{aligned}
\frac{\omega_{C}}{\Gamma} & =\frac{q B}{c m n \sigma v}=\frac{4 \pi q B r^{2}}{c \sigma \dot{M}} \\
& \approx 10^{4}\left(\frac{B}{1 \mathrm{G}}\right)\left(\frac{\sigma}{2 \times 10^{-13} \mathrm{~cm}^{2}}\right)^{-1}\left(\frac{\dot{M}}{10^{10} \mathrm{~g} \mathrm{~s}^{-1}}\right)^{-1} .
\end{aligned}
$$

Although the field strength decreases with distance from the planet, Equation (4) shows that the frequency ratio scales like $B r^{2}$. Since the dipole field strength $B \propto 1 / r^{3}$, the ratio $\omega_{C} / \Gamma$ decreases as one power of the radius and hence formally exceeds unity out to a radius $r_{C} \sim 10^{4} R_{P}$, well beyond the launching radius $r_{s}$ of the wind (where $r_{s} \approx 3 R_{P}$; see Section 4 ). This estimate for $r_{C}$ is much larger than the radial scale where the stellar environment - including the stellar wind, magnetic field, and gravity-dominates that of the planet. Although the number density of the wind may continue to decrease in this regime, the magnetic field strength will be larger than the scaling used here and the wind will remain tied to the field. We thus conclude that $\omega_{C} \gg \Gamma$ for the regime of parameter of interest and that the outflow is well coupled to the magnetic field.

Another necessary condition for the magnetic field to guide the outflow is that the magnetic pressure must be larger than the ram pressure of the flow. Here, we find the radius where the two pressures are equal, i.e., where $\rho v^{2} \approx B^{2} / 8 \pi$. We can write the density in terms of the outflow rate $\dot{M} \approx 4 \pi r^{2} \rho v=\mathcal{F}_{\mathrm{m}} 4 \pi r^{2} \rho a_{s}$, where $a_{s}$ is the sound speed and the second equality defines the parameter $\mathcal{F}_{\mathrm{m}}$. The magnetic pressure and the ram pressure are equal when $B^{2} r^{2}=2 M a_{s} \mathcal{F}_{\mathrm{m}}$. After scaling the magnetic field using the usual dipole relationship, so that $B=B_{P}\left(R_{P} / r\right)^{3}$, we can solve for the radius within which the magnetic field is 
dominant, i.e.,

$$
\begin{aligned}
\frac{r}{R_{P}} & =\left(B_{P} R_{P}\right)^{1 / 2}\left(2 \dot{M} a_{s} \mathcal{F}_{\mathrm{m}}\right)^{-1 / 4} \\
& \approx 26\left(\frac{B_{P}}{10 \mathrm{G}} \cdot \frac{R_{P}}{10^{10} \mathrm{~cm}}\right)^{1 / 2}\left(\frac{\dot{M}}{10^{10} \mathrm{~g} \mathrm{~s}^{-1}} \cdot \frac{a_{s}}{10 \mathrm{~km} \mathrm{~s}^{-1}}\right)^{-1 / 4} .
\end{aligned}
$$

This equation is evaluated using the surface field $B_{P}=10 \mathrm{G}$, the value estimated for extrasolar planets (Christensen et al. 2009; see also TAL); for comparison, the surface field strength for Jupiter is somewhat lower, $B_{P}=4.2 \mathrm{G}$ (Stevenson 2003). This result implies that the magnetic pressure exceeds the ram pressure of the outflow for radii near the planet, where "near" is defined to be within about $26 R_{P}$. However, the stellar magnetic field is generally stronger than the planetary field at these radii, so that the magnetic field pressure dominates at all distances from the planet. In addition, the sonic transitions take place at $r \sim 3 R_{P}$, well within the boundary defined by Equation (5). Using the dipole scaling relation for the field strength, we find that the magnetic pressure is larger than the ram pressure of the outflow by a factor of $\sim 10^{4}$ at the sonic surface and by a factor of $\sim 10^{6}$ at the planetary surface. As a result, the outflow must be magnetically controlled. For completeness, we note that these field configurations contain an X-point, a location where the field vanishes in the equatorial plane and where the condition (5) for magnetic pressure domination fails; this complication affects only a few streamlines and reduces the overall mass outflow rate by a small amount.

The star supports a stellar wind that also provides a ram pressure. In order for the planet to successfully launch an outflow under magnetically controlled conditions, the ram pressure of the stellar wind cannot be too large. The ratio of the ram pressure of the stellar wind to that of the planetary outflow can be written in the form

$$
\frac{P_{\mathrm{ram} *}}{P_{\mathrm{ram} P}}=\frac{(\dot{M} v)_{*}}{(\dot{M} v)_{P}}\left(\frac{r}{a}\right)^{2},
$$

where $r$ is the radial coordinate centered on the planet and the stellar parameters are evaluated at the location of the planet (a distance $a$ from the star). If the stellar wind has an outflow rate $\dot{M}_{*}=10^{12} \mathrm{~g} \mathrm{~s}^{-1}$ and outflow speed $v_{*}=400 \mathrm{~km} \mathrm{~s}^{-1}$, comparable to the values for the Sun, then the ratio of ram pressure in Equation (6) is close to unity within a few planetary radii. As shown above, $B^{2} \gg \rho v^{2}$ for the planetary wind, so that the same is true for the stellar wind; as a result, the magnetic pressure near the planet will be much larger than the ram pressure of both the stellar wind and the planetary wind, and the outflow will be magnetically controlled. At large distances from the planet, as determined from Equation (5), the stellar wind pressure will play an important role.

The star also has a magnetic field that must be taken into account. If the surface strength of the stellar field is $B_{*}$, the strength at the location of the planet will be $B \approx B_{*}\left(R_{*} / a\right)^{3}$, where $a$ is the semimajor axis of the planetary orbit. This expression would be exact in the limiting case of a pure dipole field with the planetary orbit in the equatorial plane of the dipole. In practice, however, the stellar field will be more complicated due to the stellar wind. For solar-type conditions, the field lines are closed out to radii $\sim 3 R_{*}$, but they spiral outward at larger distances. The planet radius $R_{P} \sim 10^{10} \mathrm{~cm}$ is much smaller than both the stellar radius $R_{*} \sim 10^{11} \mathrm{~cm}$ and the distance to the star $a \sim 10^{12} \mathrm{~cm}$. As a result, to leading order, the stellar contribution to the field can be considered to have constant field strength and constant direction over the region where the planetary outflow is launched; it is straightforward to show that this approximation results in error terms of order $\mathcal{O}(r / a) \sim 10^{-2}$. At the planetary surface, the ratio of the stellar field strength to that of the planet is given by

$$
\beta \equiv \frac{B(a)}{B_{P}} \approx \frac{B_{*}}{B_{P}}\left(\frac{R_{*}}{a}\right)^{3},
$$

where $B_{*}$ is the field strength on the stellar surface and $B(a)$ is the stellar field strength evaluated at the position of the planet. For the regime of parameter space of interest, this field geometry has an X-point, a location where the magnetic field vanishes (Shu 1992). Here, the stellar field is (nearly) constant in the vicinity of the planet, whereas the planetary field decreases as $B \propto 1 / r^{3}$. To leading order, the radius of the $\mathrm{X}$-point is given by

$$
r_{X} \approx \frac{a R_{P}}{R_{*}}\left(\frac{B_{P}}{B_{*}}\right)^{1 / 3} \sim 10 R_{P} .
$$

For comparison, note that the Hill radius is given by $r_{H}=$ $a\left(M_{P} / 3 M_{*}\right)^{1 / 3}$. For a Jovian planet and a solar-type star, $r_{H} \approx 0.07 a \sim 7 R_{P}$. As a result, the sphere of gravitational influence of the planet (determined by $r_{H}$ ) and the sphere of magnetic influence of the planet (determined by $r_{X}$ ) are approximately the same.

To illustrate the magnetic field geometries that arise, we plot a representative collection of magnetic field lines in Figure 1. This plot uses $\beta=0.0014$, a value that occurs (for example) when the star and the planet have comparable surface field strengths and the length scales $R_{*} / R_{P}=10$ and $a / R_{P}=100$. Magnetic field lines that originate from small polar angles on the planet surface curve off to large distances. These field lines will, in general, connect up with stellar field lines, which either end on the star or are carried out to large distances by the stellar wind. Field lines that start at lower latitudes (closer to the planetary equator) curve back and end on the planetary surface, i.e., they are closed. For a given field configuration, a well-defined fraction $F_{\mathrm{AP}}$ of the planetary surface is exposed by having its field lines open (relative to the planet). The field line that passes through the $\mathrm{X}$-point, shown by the dashed curve in Figure 1, delineates the boundary between the closed and open field lines.

In principle, currents produced by the flow could modify the planetary magnetic field, assumed here to have a nearly dipole form. This effect has been extensively studied, primarily in the context of stellar winds (e.g., Mestel 1968; Mestel \& Spruit 1987; see also TAL for an application to extrasolar planet magnetospheres). As shown in the aforementioned work, currents $J_{\perp}$ that run perpendicular to the flow can arise from either vorticity or the variations of the Bernoulli constant across field lines. These currents give rise to perturbations in the magnetic field strength, $B_{\perp}$, which are of order

$$
\frac{B_{\perp}}{B} \sim \frac{4 \pi \rho v^{2}}{B^{2}} .
$$

As shown above, this ratio is quite small within the sonic surface, typically less than $10^{-4}$, so that the additional fields produced by perpendicular currents can be neglected.

Magnetic fields can also be generated by current sheets, which arise at the interface between the field lines that carry outflowing material and those that do not. The difference in flow velocity across this boundary implies a pressure difference (from the 


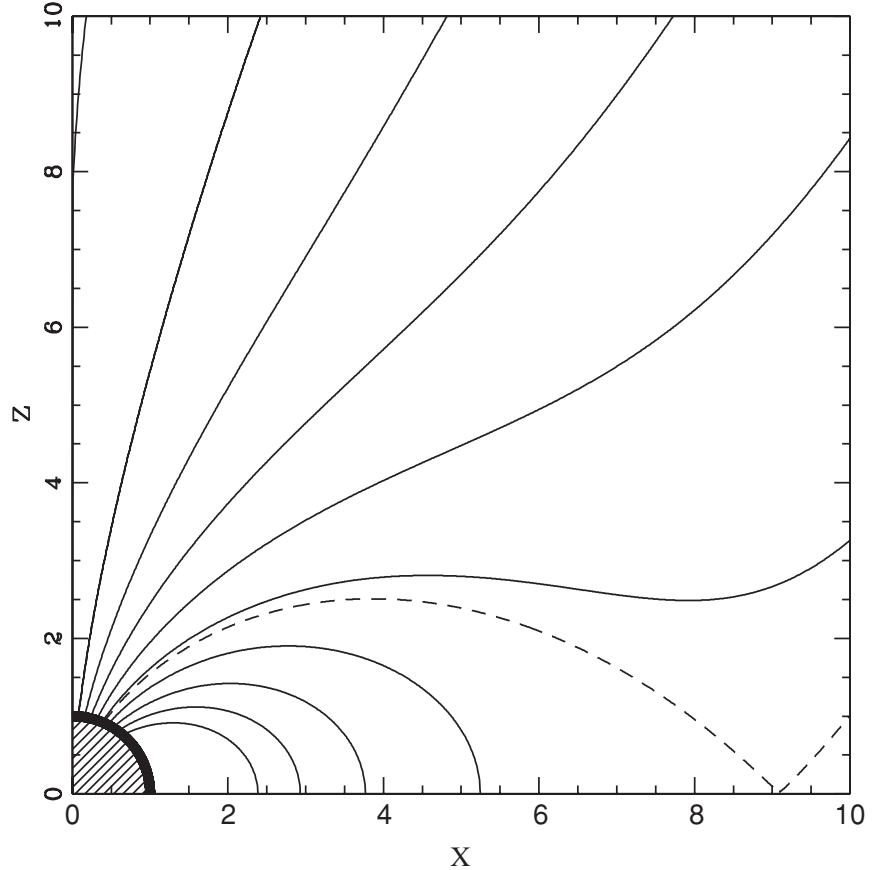

Figure 1. Magnetic field lines centered on the planet for field configurations with background parameter $\beta \approx 0.0014$. The solid curves show a set of field lines for starting values of $\sin \theta$ that are evenly spaced. The dashed curve shows the field line that passes through the X-point. The field lines near the poles are open and join onto the background field lines provided by the star and stellar wind. The field lines near the equator are closed and end back on the planetary surface. In this coordinate system, distances are measured in units of the planet radius, and the star is located at position $(x, y, z)=(100,0,0)$.

Bernoulli equation) which results in a perturbation $\delta B$ of the magnetic field. Applied to the present problem, the results of TAL imply that $(\delta B) / B \sim 8 \pi P / B^{2}$, where $P$ is the thermal pressure and $B$ is the dipole field of the planet. Over most of the parameter space of interest, the field produced by current sheets $(\delta B)$ is thus the same order as that produced by perpendicular currents $\left(B_{\perp}\right.$, see Equation (9)), and hence can be neglected to leading order $\left([\delta B] / B \sim 10^{-4}\right)$.

Perpendicular currents and current sheets can be neglected when the pressure $P \ll B^{2} /(8 \pi)$. In the case of stellar winds, this condition is violated sufficiently far from the stellar surface; at these large distances currents cannot be ignored, as they lead to open field lines, which are necessary for winds to escape (Mestel $\&$ Spruit 1987). In the present case, however, an external field allows for open field lines (relative to the planet) even in the absence of currents. As shown in Section 3.2, as long as the external field is nonzero, some of the field lines will be open; the fraction of the planetary surface that is covered by open field lines is an increasing function of the external field strength (see Equation (27)).

The above discussion defines the expected planet radius $R_{P}, \mathrm{X}$-point radius $r_{X}$, Hill's radius $r_{H}$, stellar radius $R_{*}$, and semimajor axis $a$. The principal calculation of this paper determines the radius $r_{s}$ of the sonic point, the location where the outflow is launched and can escape the planet, and finds $r_{s} \sim 3-4 R_{P}$ (Section 4). The remaining length scale in the problem is the scale height $H$ of the planetary atmosphere, where $H=k T /(\mu g)=k T R_{P} /\left(\mu G M_{P}\right)$. As a reference point, we evaluate the scale height at the atmospheric level where most of the stellar light is absorbed. At this layer, previous work (e.g., MCM) shows that the effective temperature of the planet
$T \sim 1000 \mathrm{~K}$ and hence $H \sim R_{P} / 100$; the scale height increases to $H \sim R_{P} / 10$ in the upper atmosphere where the UV photons are absorbed and $T \sim 10^{4} \mathrm{~K}$. With this specification, the length scales involved in planetary outflows obey the ordering

$$
H \ll R_{P}<r_{s}<r_{H} \sim r_{X} \sim R_{*} \ll a .
$$

The sphere of gravitational influence of the planet (from the Hill radius $r_{H}$ ) and the sphere of magnetic influence (from the $\mathrm{X}$-point radius $r_{X}$ ) are roughly the same size, and are comparable to the stellar radius. The sonic surface typically lies at a few planetary radii and is thus well inside both $r_{H}$ and $r_{X}$. On the other hand, the sphere of influence of the planet is much smaller than the star-planet distance (the semimajor axis $a$ ). This separation of scales allows for the launch of the outflow to be considered independently of the subsequent propagation of the flow.

\section{MAGNETIC FIELD GEOMETRY WITH CONSTANT BACKGROUND}

To gain further understanding of this problem and to simplify the calculations, we use the method of matched asymptotic expansions. Specifically, we divide the problem into two regimes: (1) the region near the planet where the outflow is launched, and (2) the region "far" from the planet where the magnetic field structure is determined by the field of the star (modified by the stellar wind). In the near region, the magnetic field is primarily determined by the dipole field of the planet, but nonetheless contains a contribution from the stellar field. However, this stellar contribution is nearly uniform. Exploiting this property, we can model the magnetic field near the planet through the reduced form

$$
\mathbf{B}=B_{P}\left[\xi^{-3}(3 \cos \theta \hat{r}-\hat{z})+\beta \hat{z}\right],
$$

where $\xi=r / R_{P}$ and $\beta$ is defined by Equation (7). For simplicity, we have taken the background field to point in the $\hat{z}$-direction. Notice that this reduced field is axisymmetric, so that the problem becomes two dimensional. In addition, the field is antisymmetric with respect to reflections across the $z=0$ plane; since the sign of the field does not affect the dynamics, the outflow is the same for both hemispheres of the planet.

In this initial treatment, the dipole field of the planet is augmented by a constant background field (due to the star). The stellar field is expected to be dipolar near the stellar surface, but will be modified by the stellar wind. This stellar wind acts to straighten the field lines, making them more radial. In any case, the stellar field is expected to be nearly constant, in both strength and direction, over the region where the wind is launched. However, the background field will not necessarily point in the $\hat{z}$-direction of the planetary dipole (as assumed here). Notice also that the planetary spin, and hence the planetary dipole, does not necessarily line up with direction of the orbital angular momentum, i.e., the dipole also has (in general) an arbitrary direction (e.g., Fabrycky \& Winn 2009). This paper considers the simplest case where the dipole and the background field are aligned (Equation (11)). Despite its simplicity, this ansatz displays the key features of the expected magnetic configuration: a dipole form near the planet, an (effectively) straight geometry far from the planet, and some open field lines even in the absence of currents. The more general case, where the two components lie at an arbitrary angle, should be considered in future work. In addition, if the star rotates more slowly than the angular velocity of the planetary orbit, the field lines will tend to wrap up into spiral configurations (Parker 1958); this effect should also be included in future studies. 


\subsection{Construction of the Coordinate System}

With this configuration, the magnetic field is current free and curl free, and hence can be written as the gradient of a scalar field. We define an analogous scalar field $p$ that serves as the first field of the coordinate system, i.e.,

$$
p=\left(\beta \xi-\xi^{-2}\right) \cos \theta .
$$

The gradient $\nabla p$ defines a vector field that points in the direction of the magnetic field (and hence points in the direction of the outflow). Next, we construct the perpendicular vector field $\nabla q$, where the second scalar field $q$ provides the second coordinate and is given by

$$
q=\left(\beta \xi^{2}+2 / \xi\right)^{1 / 2} \sin \theta
$$

The pair $(p, q)$ thus represents a set of perpendicular coordinates in the poloidal plane, and these can be used instead of the original spherical coordinates $(\xi, \theta)$ or the Cartesian coordinates $(x, z)$. In this version of the problem, the field is axisymmetric about the $\hat{z}$-axis, so we can use the usual azimuthal coordinate $\phi$ as the third scalar field of the set (the magnetic field is poloidal, with no toroidal component). Note that both $p$ and $q$ are dimensionless; one can reinsert factors of the planetary radius $R_{P}$ (as necessary) to convert back to physical units. Notice also that in the limit $\beta \rightarrow 0$, one recovers the coordinates for a dipole (Radoski 1967).

The set of covariant basis vectors $\underline{\epsilon}_{j}$ arises from the gradients of the scalar fields that define the coordinates. If we express these basis vectors in terms of the original spherical coordinates $(\xi, \theta, \phi)$, the basis takes the form

$$
\begin{gathered}
\underline{\epsilon}_{p}=\left(\beta+2 \xi^{-3}\right) \cos \theta \hat{r}-\left(\beta-\xi^{-3}\right) \sin \theta \hat{\theta} \\
\epsilon_{q}=\left(\beta+2 \xi^{-3}\right)^{-1 / 2}\left[\left(\beta-\xi^{-3}\right) \sin \theta \hat{r}+\left(\beta+2 \xi^{-3}\right) \cos \theta \hat{\theta},\right],
\end{gathered}
$$

and

$$
\underline{\epsilon}_{\phi}=\frac{1}{\xi \sin \theta} \hat{\phi}
$$

The quantities $(\hat{r}, \hat{\theta}, \hat{\phi})$ are the usual unit vectors for spherical coordinates. However, one should keep in mind that $\underline{\epsilon}_{j}$ are basis vectors (not unit vectors), so that their length is not, in general, equal to unity (for further discussion, e.g., see Weinreich 1998). In general, the corresponding scale factors are given by the relation

$$
h_{j}=\left|\underline{\epsilon}_{j}\right|^{-1},
$$

so that the scale factors for this coordinate system can be written in the form

$$
\begin{gathered}
h_{p}=\left[\left(\beta+2 \xi^{-3}\right)^{2} \cos ^{2} \theta+\left(\beta-\xi^{-3}\right)^{2} \sin ^{2} \theta\right]^{-1 / 2}, \\
h_{q}=\left(\beta+2 \xi^{-3}\right)^{1 / 2}\left[\left(\beta+2 \xi^{-3}\right)^{2} \cos ^{2} \theta+\left(\beta-\xi^{-3}\right)^{2} \sin ^{2} \theta\right]^{-1 / 2},
\end{gathered}
$$

and

$$
h_{\phi}=\xi \sin \theta .
$$

The general form of the divergence operator is thus given by

$\nabla \cdot \mathbf{V}=\frac{1}{h_{p} h_{q} h_{\phi}}\left[\frac{\partial}{\partial p}\left(h_{q} h_{\phi} V_{p}\right)+\frac{\partial}{\partial q}\left(h_{p} h_{\phi} V_{q}\right)\right]+\frac{1}{\xi \sin \theta} \frac{\partial V_{\phi}}{\partial \phi}$.
In this problem, the fields are axisymmetric so that the $\phi$ derivatives vanish. Further, for flow along field lines, the vector fields (e.g., the velocity field) have only one component and depend on only one coordinate, so that the divergence operator collapses to the form

$\nabla \cdot \mathbf{V}=\frac{1}{h_{p} h_{q} h_{\phi}} \frac{\partial}{\partial p}\left(h_{q} h_{\phi} V_{p}\right)=\frac{1}{h_{p}} \frac{\partial V_{p}}{\partial p}+\frac{V_{p}}{h_{p} h_{q} h_{\phi}} \frac{\partial}{\partial p}\left(h_{q} h_{\phi}\right)$.

For convenience, we define the following ancillary functions:

$f \equiv \beta+2 \xi^{-3}, \quad g \equiv \beta-\xi^{-3}$, and $H \equiv f^{2} \cos ^{2} \theta+g^{2} \sin ^{2} \theta$.

In terms of these functions, we note that $\left(h_{q} h_{\phi}\right)=q H^{-1 / 2}$ and that $|\mathbf{B}|^{2}=H B_{P}^{2}$.

This specification of the divergence operator is implicit. One could invert Equations (12) and (13), and then write the spherical coordinates $(\xi, \theta)$, the scale factors $\left(h_{p}, h_{q}, h_{\phi}\right)$, and the ancillary functions $(f, g, H)$ as functions of the new coordinates $(p, q)$. However, the definitions of $(p, q)$ are cubic functions of $(\xi, \theta)$, so that the solutions of the cubic inversion are complicated and unwieldy (but still can be written down analytically). For clarity, we leave this construction in implicit form.

\subsection{Fractional Active Area of the Planetary Surface}

With the field configuration of Equation (11), some fraction $F_{\text {AP }}$ of the magnetic field lines that originate on the planetary surface are open, whereas some field lines curve back onto the planet. Using this simplified model for the magnetic field, we can determine the fraction $F_{\mathrm{AP}}$. In this context, the open field lines that continue to "infinity" in this reduced problem join onto the magnetic field lines of the star and stellar wind (although the subsequent curvature of the field lines is not captured by this model). One can define a benchmark mass outflow rate to be that obtained for spherical flow over the entire planetary surface. We note that the fraction $F_{\mathrm{AP}}$ of the surface that supports open field lines is related to-but is not equivalent to- the fraction of this benchmark outflow rate that the planet produces (see Section 4.4).

The first step is to solve for the magnetic field lines. For this field configuration, this construction can be done analytically. Assume that a field line begins at coordinates $\left(\xi=1, \theta_{0}\right)$ corresponding to the planetary surface. The field line can then be represented as a curve in the plane such that

$$
\left(\frac{\sin \theta}{\sin \theta_{0}}\right)^{2}=\xi \frac{2+\beta}{2+\beta \xi^{3}}
$$

Note that this expression is equivalent to the statement $q=$ $\sin \theta_{0}(2+\beta)^{1 / 2}=$ constant; this result must hold since the coordinate $q$ was constructed so that $\nabla q$ is perpendicular to the field lines. Using this solution, we can solve for the angular coordinate $\theta_{X}$ for the field line that goes through the X-point. In this case, the $\mathrm{X}$-point radius $\xi_{X}$ is given by the condition

$$
\beta \xi_{X}^{3}=1
$$

Note that this result is exact for the reduced field configuration considered here. The critical angle $\theta_{X}$ is given by

$$
\sin ^{2} \theta_{X}=3 \beta^{1 / 3} /(2+\beta),
$$


where this expression is valid for $\beta \leqslant 1$. The field lines that originate at small angles, $0 \leqslant \theta \leqslant \theta_{X}$, with $\theta_{X}$ defined above, are those that are open (reach spatial infinity) in this reduced problem. In the full problem, these open field lines join onto the background field lines of the star and stellar wind. The resulting fraction $F_{\mathrm{AP}}$ of the surface area of the planet that can support an outflow is then given by

$$
F_{\mathrm{AP}}=1-\left[1-\frac{3 \beta^{1 / 3}}{2+\beta}\right]^{1 / 2}
$$

Note that for $\beta \geqslant 1$, the background (stellar) field dominates that of the planet, field lines originating from all planetary latitudes are open, and hence $F_{\mathrm{AP}}=1$. In the opposite limit of small $\beta \ll 1$, the fraction $F_{\mathrm{AP}} \approx 0.75 \beta^{1 / 3}$; since $\beta \sim 10^{-3}$ for typical cases, only about $10 \%$ of the planet surface can support outflow.

With this field geometry, the field lines become asymptotically straight, so that the outflow has a cylindrical form in the limit $\xi \rightarrow \infty$. The outer surface of the resulting outflow cavity is delineated by the field line that passes through the X-point. If we use Equation (24) for the critical streamline and then take the limit $\xi \rightarrow \infty$, we find that the radius $\varpi_{\infty}$ of this cylinder is given by

$$
\varpi_{\infty}=\sqrt{3} r_{X}=\sqrt{3} \beta^{-1 / 3} R_{P}=\sqrt{3}\left(B_{P} / B_{*}\right)^{1 / 3}\left(a R_{P} / R_{*}\right) .
$$

This cylindrical flow represents the outer limit of the inner problem, the regime where the outflow is launched from the planetary surface. This flow also represents the inner limit of the outer problem, the regime where the flow follows the stellar field lines. Note that for typical parameters $\varpi_{\infty} \approx 10 R_{P} \approx R_{*}$.

Since the magnetic field strength vanishes along the critical streamline, the magnetic pressure will not be strong enough to dominate the ram pressure near the X-point. We can estimate the fraction of streamlines that are affected by this issue: consider the surface where $\xi=\xi_{X}=\beta^{-1 / 3}$. For a given angle $\theta$ on this surface, the magnetic field strength (from Equation (11)) is given by $B=3 B_{P} \beta \cos \theta$. For the flow to remain magnetically controlled, the field strength must be larger than a critical value $B_{C}$ determined by the ram pressure of the outflow $\left(B_{C}^{2}=8 \pi \rho v^{2}\right)$, where we expect $B_{C} \approx 0.001 \mathrm{G}$ for typical cases (see Equation (5)). This condition requires $\cos \theta>B_{C} /\left(3 B_{P} \beta\right)$, which in turn restricts the coordinate $q$ that specifies the streamlines to the range defined by

$$
q^{2}<\left[1-\left(\frac{B_{C}}{3 B_{P} \beta}\right)^{2}\right] q_{X}^{2}
$$

Since we expect $B_{C} \sim \beta B_{P}$, the range of streamlines that are unaffected by the X-point issue is roughly given by $q<$ $q_{X} \sqrt{8} / 3$. In other words, for $\sim 94 \%$ of the streamlines, the flow remains magnetically controlled over the entire space. For the remaining $\sim 6 \%$, the flow is magnetically controlled except for a small region (of size $(\Delta L) \sim r_{X} / 3$ ) surrounding the X-point. Note that outflow can still take place along these streamlines, but that the flow pattern will be slightly different than that given by the unperturbed magnetic field geometry. For the remainder of this paper, for simplicity, we consider the flow to take place over the full range of open streamlines $q \leqslant q_{X}$, while noting that this approximation may overestimate the outflow rates by a few percent.

\section{OUTFLOWS FROM PLANETARY SURFACES}

Given the specification of the magnetic field structure and hence the flow geometry (Sections 2 and 3), we now consider the launch of a wind or outflow from the surface of the planet. After writing down the full set of equations of motion, we consider a reduced version of the problem where the flow is taken to be isothermal. For this case, the solutions for the dimensionless fluid fields can be found analytically, including the required conditions for the flow to pass smoothly through the sonic transition. In order to complete the solution, we must then specify the values for the physical parameters, i.e., the density $\rho_{1}$ at the inner boundary and the sound speed $a_{s}$ (taken to be constant).

\subsection{Formulation of the Wind/Outflow Problem}

The equations of motion for this problem include the continuity equation

$$
\frac{\partial \rho}{\partial t}+\nabla \cdot(\rho \mathbf{u})=0
$$

the force equation

$$
\frac{\partial \mathbf{u}}{\partial t}+\mathbf{u} \cdot \nabla \mathbf{u}=-\nabla \Psi-\frac{1}{\rho} \nabla P+\frac{1}{4 \pi \rho}(\nabla \times \mathbf{B}) \times \mathbf{B},
$$

the energy equation

$$
\rho\left(\frac{\partial \mathcal{E}}{\partial t}+\mathbf{u} \cdot \nabla \mathcal{E}\right)=-P \nabla \cdot \mathbf{u}+\Gamma-\Lambda,
$$

and the evolution equation for the magnetic field

$$
\frac{\partial \mathbf{B}}{\partial t}+\nabla \times(\mathbf{B} \times \mathbf{u})=-\nabla \times\left(\eta_{\mathrm{res}} \nabla \times \mathbf{B}\right),
$$

where $\eta_{\text {res }}$ is the resistivity. This paper considers the magnetic fields to be fixed and strong enough not to be changed by the outflow. In a full treatment, however, the back-reaction of the outflow on the magnetic field should be taken into account.

In this paper, we consider the gravitational potential $\Psi$ to be that of the planet, which is taken to be spherical with mass $M_{P}$ and radius $R_{P}$. Since the planet orbits the star, the full potential has an additional contribution from the rotating frame of reference. The order of this correction term is $\mathcal{O}\left(M_{*} r^{3} / M_{P} a^{3}\right)$, so that it has size $\sim 10^{-3}$ near the planet surface and size $\sim 0.03$ near the sonic surface. As a result, this term does not greatly affect the launch of the outflow and is not included here. To consistent order, we also ignore the tidal forces from the stellar gravitational field (note that the sonic surface is well inside the Roche radius). We thus work in the regime where the gravitational force is dominated by that of the planet.

In the energy Equation (32), $\mathcal{E}$ is the specific energy of the fluid, $\Gamma$ is the heating rate (per unit volume), and $\Lambda$ is the cooling rate. The heating is primarily due to UV flux from the central star (e.g., see MCM and references therein) so that $\Gamma$ can be written in the form

$$
\Gamma=\eta_{\mathrm{abs}} F_{\mathrm{Uv}} e^{-\tau} \sigma_{\mathrm{uv}} n_{0},
$$

where $\eta_{\text {abs }}$ is the fraction of the UV energy that is deposited by heat, $\sigma_{\mathrm{uv}}$ is the cross section for UV photons, $F_{\mathrm{UV}}$ is the unattenuated UV flux of the star at the location of the planet, and $\tau$ is the optical depth (from the star to the point where the heating term is evaluated). 
The cooling process is primarily due to $\operatorname{Ly} \alpha$ radiation that is emitted by Hydrogen atoms as they are excited via collisions (Black 1981). To leading order, the cooling term can be represented by a function of the form

$$
\Lambda \approx C n_{0} n_{+} e^{-T_{C} / T},
$$

where $C=7.5 \times 10^{-19}, T_{C}=118,348 \mathrm{~K}$; the resulting cooling rate has units of erg $\mathrm{cm}^{-3} \mathrm{~s}^{-1}$. Note that this particular form is only valid for temperatures $T<12,000 \mathrm{~K}$; at higher temperatures the gas cools even more efficiently (Spitzer 1978). In any case, the cooling rate is large enough that the gas temperature never increases beyond an effective maximum $T_{\max } \approx 10^{4} \mathrm{~K}$. In order for Ly $\alpha$ radiation to act as the primary cooling mechanism, it must dominate over other processes and the radiation must be able to escape; both of these conditions are met, as shown in the appendices of MCM.

Since the heating and cooling rates depend on the state of ionization, through the number densities of the neutrals $n_{0}$ and ions $n_{+}$, we also need the equation of ionization balance:

$$
\frac{\partial n_{+}}{\partial t}+\nabla \cdot\left(\mathbf{u} n_{+}\right)=\frac{F_{\mathrm{UV}}}{\langle h \nu\rangle} e^{-\tau} \sigma_{\mathrm{uv}} n_{0}-\alpha_{R} n_{+}^{2},
$$

where the recombination coefficient $\alpha_{R} \approx 2.7 \times 10^{-13}$ $\left(T / 10^{4} \mathrm{~K}\right)^{-0.9} \mathrm{~cm}^{3} \mathrm{~s}^{-1}$ (Storey \& Hummer 1995). For the remaining parameters, we take $\sigma_{\mathrm{uv}}=2 \times 10^{-18} \mathrm{~cm}^{2}$ and $\langle h \nu\rangle=$ $2.2 \times 10^{11}$ erg (e.g., Spitzer 1978).

Note that the planet is only heated on the side facing the star, whereas cooling takes place over the entire surface of the planet. This work implicitly assumes that the UV heating is distributed uniformly throughout the upper atmosphere of the planet. This approximation, in turn, is valid when zonal winds are strong enough to provide the required redistribution. Although the issue is not settled, current models suggest that strong winds are present, so that uniform heating is a reasonable approximation (for further discussion, e.g., see Batygin \& Stevenson 2010; Langton \& Laughlin 2008, and references therein).

As formulated here, Equations (30)-(36) make up a compete set that can be solved for the fluid fields. However, even if we use a simplified magnetic field geometry, with the coordinates constructed in Section 3, the problem remains intrinsically three dimensional: Although the flow is axisymmetric and follows the coordinate $p$ defined by Equation (12), the heating comes from the central star which lies off to one side. As a result, the incoming photons do not follow the coordinates, and a full solution for the heating/cooling of the outflow requires one to solve a three-dimensional radiative transfer problem. Before embarking on that task, it is useful to have solutions for an approximate treatment. Toward this end, a simplified version of the problem is formulated in the next section.

\subsection{Reduced Equations of Motion}

In this section, we consider steady-state solutions and assume that the magnetic field structure due to the planet (and the star) is strong enough to dominate the flow. As a result, in this regime, the magnetic field is fixed and current free. The continuity, force, and induction equations thus reduce to the forms

$$
\nabla \cdot(\rho \mathbf{u})=0, \quad \mathbf{u} \cdot \nabla \mathbf{u}+\nabla \Psi+\frac{1}{\rho} \nabla P=0, \quad \text { and } \quad \mathbf{B}=\Upsilon \rho \mathbf{u},
$$

where the parameter $\Upsilon$ is constant along streamlines (e.g., Shu et al. 1994; Cai et al. 2008).
The velocity vector $\mathbf{u}$ follows the magnetic field lines, which follow the coordinate $p$ in the system constructed in Section 3. In other words, the flow velocity has only one component, which points in the direction of the magnetic field $\hat{p}=h_{p} \underline{\epsilon}_{p}$ (by construction). Next, we assume that the flow is isothermal with constant sound speed $a_{s}$ and define the following dimensionless quantities:

$$
u=u(p) \equiv \frac{|\mathbf{u}|}{a_{s}}, \quad \alpha \equiv \frac{\rho}{\rho_{1}}, \quad \xi \equiv \frac{r}{R_{P}}, \quad \text { and } \quad \psi \equiv \frac{\Psi}{a_{s}^{2}} .
$$

Here, $R_{P}$ is the radius of the planet and $\rho_{1}$ is the density at the inner boundary $\xi=1$. The continuity equation thus takes the form

$$
\alpha \frac{\partial u}{\partial p}+u \frac{\partial \alpha}{\partial p}=-\frac{\alpha u}{h_{q} h_{\phi}} \frac{\partial}{\partial p}\left(h_{q} h_{\phi}\right),
$$

and the force equation becomes

$$
u \frac{\partial u}{\partial p}+\frac{1}{\alpha} \frac{\partial \alpha}{\partial p}=-\frac{\partial \psi}{\partial p}=-\frac{\partial \psi}{\partial \xi} \frac{\partial \xi}{\partial p} .
$$

These equations can be integrated immediately to obtain the solutions

$$
\alpha u h_{q} h_{\phi}=\alpha u q H^{-1 / 2}=\lambda
$$

and

$$
\frac{1}{2} u^{2}+\log \alpha+\psi=\varepsilon .
$$

Although the potential $\psi$, in general, contains additional contributions (e.g., tidal forces), we specialize to the case that includes only the gravitational potential of the planet so that $\psi=-b / \xi$, where $b \equiv G M_{P} /\left(a_{s}^{2} R_{P}\right)$. Note that the quantity $h_{q} h_{\phi}=q H^{-1 / 2}$ is proportional to the inverse of the magnetic field strength (consistent with the third part of Equation (37)). The parameters $\lambda$ and $\varepsilon$ are constant along streamlines, but are not, in general, the same for all streamlines (they are functions of $q$ ). In order for the flow to pass smoothly through the sonic point, only particular values of the constant $\lambda$ are allowed. This constraint is considered in the following section.

The boundary conditions at the planetary surface take the form

$$
\xi=1, \quad \alpha=1, \quad \text { and } \quad u=u_{1}=\lambda H_{1}^{1 / 2} / q .
$$

Since $\lambda$ is determined by the conditions at the sonic point, $u_{1}$ is specified. In addition, the remaining parameter $\varepsilon$ is determined by evaluating the force equation at the inner boundary, i.e.,

$$
\varepsilon=\frac{1}{2} u_{1}^{2}-b=\frac{\lambda^{2} H_{1}}{2 q^{2}}-b .
$$

The outflow starts with subsonic speeds so that $u_{1} \ll 1$ (below we find that $u_{1} \sim \lambda / q \sim 0.01$ ), whereas typical planet properties imply that $b \sim 10$. As a result, one can use the approximation $\varepsilon \approx-b$ with good accuracy.

\subsection{Sonic Point Conditions}

Critical points in the flow arise when the fluid speed is equal to the transport speed. In general, magnetic media support three types of MHD waves and hence allow for three types of critical points (e.g., Shu 1992). In this case, however, the flow is confined to follow the magnetic field lines, so that only one possible critical point arises, in this case where the flow speed equals 
the sound speed. For the equations of motion (41) and (42), the required matching conditions at the sonic point take the form

$$
u^{2}=1 \quad \text { and } \quad \frac{1}{h_{q} h_{\phi}} \frac{\partial}{\partial p}\left(h_{q} h_{\phi}\right)=\frac{\partial \Psi}{\partial \xi} \frac{\partial \xi}{\partial p}=\frac{b}{\xi^{2}} \frac{\partial \xi}{\partial p} .
$$

We must thus evaluate the geometrical factor $\mathcal{G}$ defined by

$$
\mathcal{G} \equiv \frac{1}{h_{q} h_{\phi}} \frac{\partial}{\partial p}\left(h_{q} h_{\phi}\right)
$$

Note that in spherical coordinates, this factor would have the usual form $2 / \xi$. In terms of the ancillary functions from Equation (23), the partial derivatives can be expressed as

$$
\frac{\partial \xi}{\partial p}=\frac{f \cos \theta}{H} \text { and } \quad \frac{\partial \theta}{\partial p}=-\frac{g \cos \theta}{\xi H},
$$

and the geometrical factor $\mathcal{G}$ takes the form

$$
\mathcal{G}=\frac{3 \cos \theta}{H^{2} \xi^{4}}\left[2 f^{2} \cos ^{2} \theta-g(g+2 f) \sin ^{2} \theta\right] .
$$

The matching condition at the sonic point can then be written in the form

$$
b=\frac{3}{f H \xi^{2}}\left[2 f^{2} \cos ^{2} \theta-g(g+2 f) \sin ^{2} \theta\right] .
$$

One can eliminate the explicit angular dependence from this expression using the result $\sin ^{2} \theta=q^{2} / \xi^{2} f$ and noting that $q$ is a constant along the direction of the flow. The right-hand side of the above equation (for given $q$ ) thus becomes a function of $\xi$ only,

$$
\frac{b}{3}=\frac{2 f^{2}-\left(g^{2} / f+2 g+2 f\right) q^{2} / \xi^{2}}{f^{3} \xi^{2}+\left(g^{2}-f^{2}\right) q^{2}} .
$$

For given planetary properties (set by the value of $b$ ), magnetic field strength ratio (set by $\beta$ ), and starting angle $\theta_{0}$ of the streamline (set by $q$ ), Equation (50) provides an algebraic expression that can be solved for the value of $\xi=\xi_{s}$ at the sonic point. With $\xi_{s}$ specified, the angle $\theta\left(\xi_{s}\right)$ is also specified, and hence the value of $p=\xi g \cos \theta$ is determined. Finally, the value of the parameter $\lambda$ that allows for smooth flow through the sonic point is given by

$$
\lambda=\lambda(q)=q H_{s}^{-1 / 2} \exp \left[\frac{\lambda^{2} H_{1}}{2 q^{2}}+\frac{b}{\xi_{s}}-b-\frac{1}{2}\right],
$$

where the subscript " $s$ " ("1") implies that the quantity is evaluated at the sonic point (inner boundary). Equation (51) provides an implicit solution for the parameter $\lambda$. However, the $\lambda^{2}$ term on the right-hand side of Equation (51) is extremely small (it is equal to $u_{1}^{2} / 2 \ll 1$ ) and can be ignored to leading order; doing so results in a direct expression for the parameter $\lambda$ (after Equation (50) has been solved to find the value of $\xi_{s}$ ). We also note that one can define an alternate parameter $\tilde{\lambda} \equiv \lambda / q$, which is useful because it is easier to find solutions for $\tilde{\lambda}$ (e.g., when $q \rightarrow 0$ ).

Figure 2 shows the sonic surface for a planet/star system with typical properties. Here, the parameter $\beta=10^{-3}$, which holds when the surface fields on the planet and the star are equal, and the semimajor axis $a=10 R_{*}$; the parameter $b=10$, which holds for planets with mass $M_{P}=0.75 M_{J}$, radius $R_{P}=$

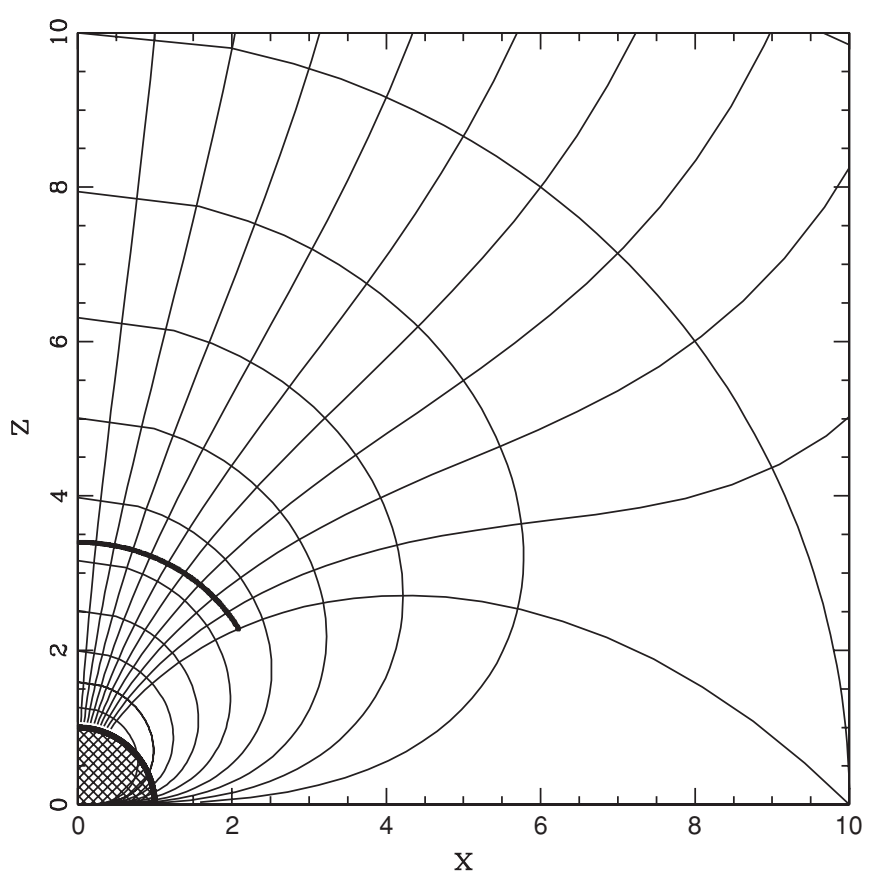

Figure 2. Sonic transition surface for the planet with dimensionless gravitational potential depth $b=G M_{P} /\left(a_{s}^{2} R_{P}\right)=10$ and background magnetic field strength parameter $\beta=10^{-3}$. The heavy solid curve near $\xi=3.3$ shows the location where the flow passes through the sonic point. The light solid curves show the underlying $(p, q)$ coordinate system. The Cartesian coordinates $x$ and $z$ are given in units of the planetary radius.

$10^{10} \mathrm{~cm}$, and sound speed $a_{s}=10 \mathrm{~km} \mathrm{~s}^{-1}$. The sonic transition occurs at nearly constant dimensionless radius $(\xi \approx 3.3)$, as delineated by the heavy curve. The light solid curves show the underlying coordinate system, i.e., the lines of constant $p$ and $q$. Only the streamlines (constant $q$ ) corresponding to open magnetic field lines are shown. The last coordinate line shown is the one passing through the X-point. For the perpendicular coordinate, the value $p=0$ corresponds to the surface passing through the X-point, and that surface is a sphere. Negative values of $p$ correspond to surfaces that are more highly curved (compared to a sphere), whereas positive values of $p$ produce flatter surfaces. Note that the sonic surface lies well within the surface passing through the X-point. As a result, the flow diverges significantly faster than that of a spherically symmetric wind.

The right-hand side of Equation (50) is not, in general, a monotonic function of $\xi$; it reaches a maximum and then decreases in the limit $\xi \rightarrow \infty$. If the maximum value is too small, then Equation (50) has no solutions, and the outflow will not pass through the sonic point within the context of this simplified model. One can show that the right-hand side of Equation (50) is an increasing function of $q$ for sufficiently small $\xi$ (in the regime where matching occurs). As a result, the minimum value occurs for the smallest value of $q$, i.e., along the pole where $q=0$. For this streamline, the matching condition is the most difficult to meet; the sonic point is thus given by solutions to the cubic polynomial $b=6 / f \xi^{2}$, or, equivalently,

$$
\beta \xi^{2}+2 / \xi=6 / b
$$

This equation has no real solutions if either parameter $\beta$ or $b$ is too large; solutions require $\beta b^{3} \leqslant 8$, or equivalently $\beta \leqslant$ $\beta_{\max }=8 / b^{3}$. Since $b \sim 10$, the value of $\beta_{\max } \sim 0.01$. Only smaller values of $\beta<\beta_{\max }$ allow for smooth outflow solutions. 


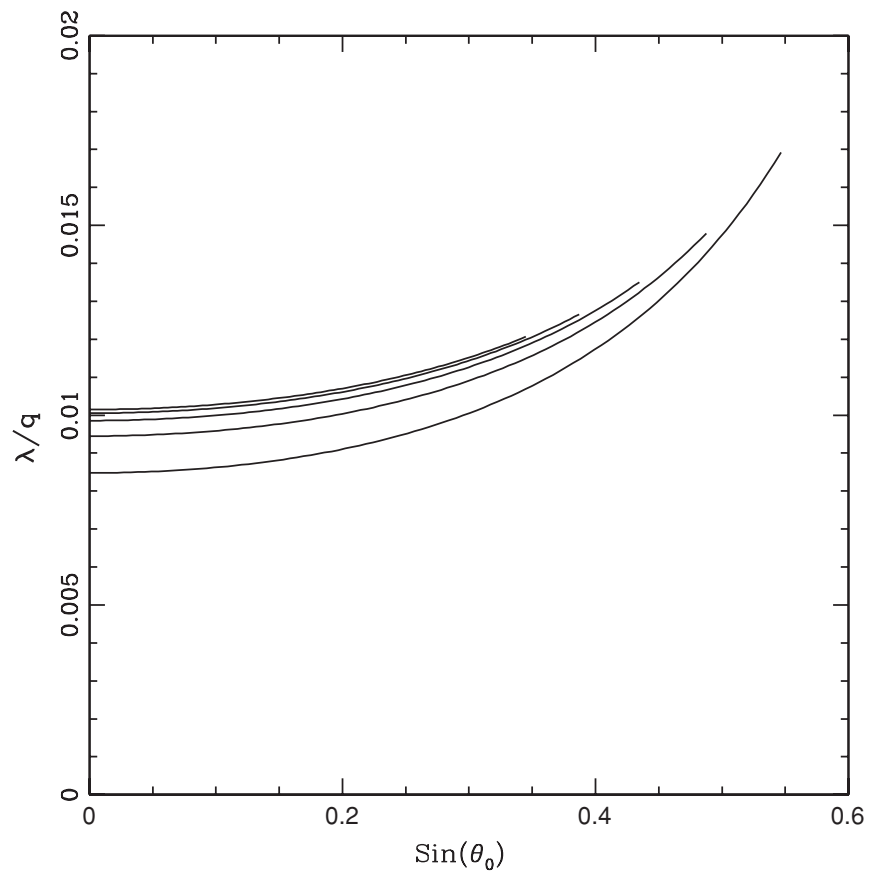

Figure 3. Values of the parameter $\lambda / q$ that allow the outflow to pass smoothly through the sonic point. The parameters $\lambda$ are shown here as a function of $\sin \theta_{0}=q(2+\beta)^{-1 / 2}$, which determines the starting point of the streamlines. All cases use $b=10$ (where $b=G M_{P} /\left(a_{s}^{2} R_{P}\right)$ sets the depth of the gravitational potential of the planet). Curves are shown for a range of the parameter that sets the relative strength of the stellar and planetary magnetic fields: $\beta=0.008$ (bottom curve), 0.004, 0.002, 0.001, and 0.0005 (top curve). For smaller values of $\beta$, the curves converge toward a well-defined locus (although the range in $\sin \theta_{0}$ shrinks).

For solutions that pass through the sonic point, so that $\lambda$ is specified, Figure 3 presents the resulting values of $\lambda / q$ over the range of allowed streamlines. These results are shown for a range of the magnetic field strength parameter $\beta$. For the planetary properties used here, where $b=10$, the maximum allowed value of $\beta=0.008$ is shown by the lowest curve in Figure 3. Larger values of $\beta$ do not allow for a sonic transition over the full range of open field lines. The resulting $\lambda / q$ for smaller values of $\beta$ converge toward the result for $\beta=0$. In addition, the range of allowed streamlines, given here by the range of allowed starting angles $\theta_{0}$, decreases with decreasing $\beta$ (see Equation (26)). Notice also that $\lambda / q$ is relatively slowly varying over the range of allowed streamlines. For the largest possible $\beta, \lambda / q$ varies by a factor of $\sim 2$ from the pole to the angle of the last open field line; the results for smaller $\beta$ show much less variation.

Note that the results derived above make sense in the limits: for flow near the planetary surface, where the magnetic field is determined by the dipole of the planet, the sonic point condition of Equation (50) reduces to the form $b=3 \xi$ (along the pole). This solution results from the continuity equation $\partial_{\xi}\left(\xi^{3} \rho u\right)=0$, where this form for the divergence operator is expected for flow that follows a dipole field. In the opposite limit where $\xi \rightarrow \infty$, the field lines and the streamlines become asymptotically straight and point in the $\hat{z}$-direction. In this limit, we recover the results for one-dimensional flow, where the continuity equation has the form $\partial_{z}(\rho u)=0$. The solutions that smoothly pass through the sonic point are those where the magnetic field of the planet dominates, so that the sonic point $\xi_{s} \approx b / 3$. Using this result, we can define a fiducial value for the parameter $\lambda / q$, i.e.,

$$
(\lambda / q)_{0}=\frac{b^{3}}{54} \exp [5 / 2-b] \approx 0.2256 b^{3} \mathrm{e}^{-b} .
$$

For expected planetary properties with $b \approx 10$, we find $(\lambda / q)_{0} \approx 0.01$. Notice also that $\lambda / q$ sets the scale for the mass outflow rate and that this quantity decreases exponentially with increasing depth of the gravitational potential well of the planet (set by $b$ ).

For solutions that smoothly pass through the sonic transition, we can also find the asymptotic speed $u_{\infty}$, i.e., the value realized in the limit $\xi \rightarrow \infty$. In this regime, the reduced equations of motion (41) and (42) are simplified further to take the forms

$$
u \alpha=\lambda \beta / q \quad \text { and } \quad \frac{1}{2} u^{2}+\log \alpha=\varepsilon,
$$

where both $\lambda$ and $\varepsilon$ are known functions of the parameter $q$ that specifies the streamline. The asymptotic speed $u=u_{\infty}$ is thus given by the expression

$$
u_{\infty}^{2}-\log u_{\infty}^{2}=2 \varepsilon+2 \log (q / \beta \lambda) .
$$

Note that Equation (55) has two roots whenever real solutions exist, which requires the right-hand side to be greater than unity. One root corresponds to $u_{\infty}^{2}<1$, whereas the (physical) root of interest corresponds to $u_{\infty}^{2}>1$.

Figure 4 shows the dimensionless velocity and density profiles for outflows characterized by different values of the constant $\lambda$. These profiles are shown for flow from the poles, so that $q=0$ and the flow direction is given by $\hat{r}=\hat{z}$. In the top panel, the central curve shows the velocity profile for the critical value of $\lambda / q$ that allows the flow to pass smoothly through the sonic point. If the value of $\lambda / q$ is too small (bottom curve), then the outflow speed never reaches the sound speed. Instead, the flow velocity reaches a maximum at the radius of the sonic point and then decreases for larger $\xi$. On the other hand, if the value of $\lambda / q$ is too large (top curve), the outflow cannot reach the sonic point in a smooth manner. This plot is thus analogous to that found for the well-known Parker wind (see Figure 1 of Parker 1965) and for Bondi-Hoyle accretion (e.g., see Shu 1992 for further discussion).

The bottom panel of Figure 4 shows the corresponding density profiles. Here, the solid curve shows the result for the critical value of $\lambda / q$ that allows for a smooth sonic transition. The supercritical and subcritical cases are shown as the dashed curves. Note that the density profile is extremely steep near the planetary surface, and then levels out at large radii $\xi$. This behavior is a reflection of the divergence operator, which follows the magnetic field lines, which in turn spread rapidly near the planet and become straight at large distances $\xi$. Notice also that the density for the subcritical case is larger than that of the critical case for $\xi \gg 1$. Although the mass outflow rate is smaller for the subcritical case (which lowers the density), the asymptotic speed (see the top panel) is much smaller and this latter effect increases the density and produces the observed behavior.

For completeness, we can find the values of the parameter $\lambda / q$ for field configurations where the matching condition (Equation (49)) at the sonic point cannot be met. In this case, the largest value of $\lambda$, and hence the largest outflow rate, occurs when the outflow velocity approaches the sound speed in the limit $\xi \rightarrow \infty$. In this case, we can combine Equations (41)-(44), 

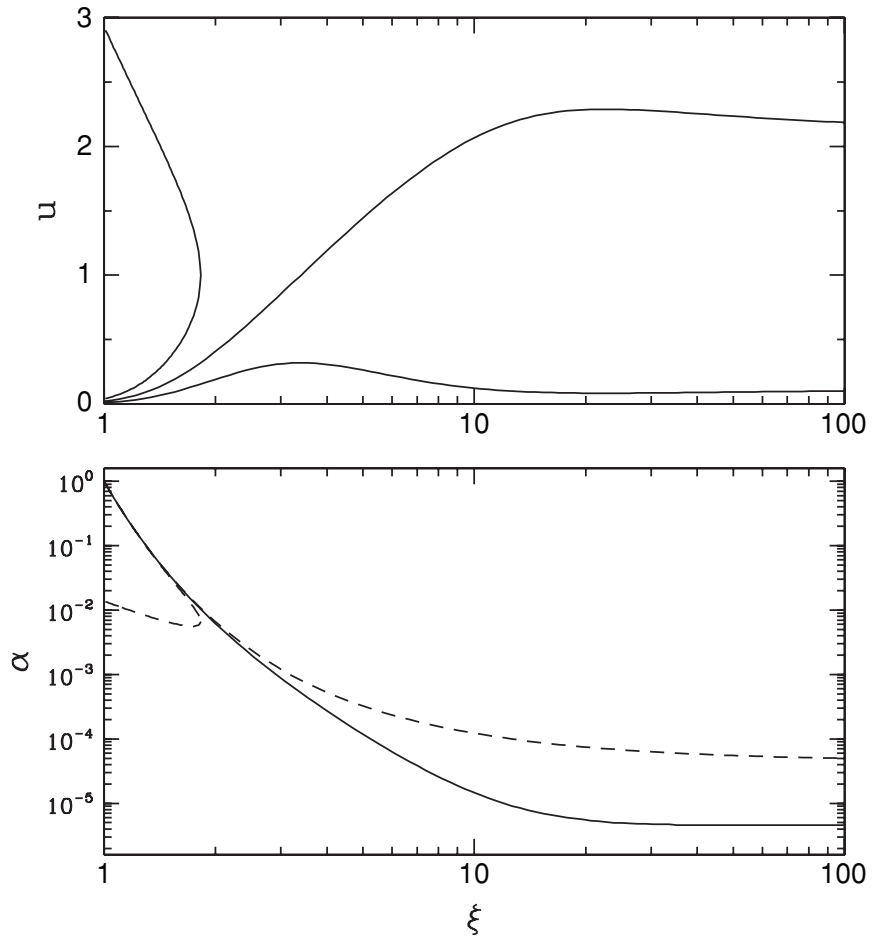

Figure 4. Top panel shows the velocity profiles for outflows from planetary surfaces for flow with $q=0$ (from the planetary pole). The three curves show the velocity as a function of radius $\xi$ for the critical value of $\lambda / q$ (central curve), for a subcritical case (bottom curve), and a supercritical case (top curve). These velocity profiles were calculated using $b=10$ and $\beta=10^{-3}$. Bottom panel shows the corresponding density profiles. The critical case is shown as the solid curve, whereas the dashed curves show the subcritical case (which continues to large $\xi$ ) and the supercritical case (which is confined to small $\xi$ ).

while setting $u \rightarrow 1$, and then solve for the value of the parameter $\lambda / q$ :

$$
\lambda / q=\beta^{-1} \exp \left[-(b+1 / 2)+\frac{\lambda^{2} H_{1}}{2 q^{2}}\right] \approx \beta^{-1} \exp [-(b+1 / 2)] .
$$

\subsection{Dimensionless Mass Outflow Rate}

In this geometry, the continuity equation reduces to the form $\alpha u q H^{-1 / 2}=\lambda=$ constant (see Equation (41)). Because the quantity $q H^{-1 / 2}$ does not have units of area, the constant $\lambda$ is not the mass outflow rate. As a result, we need to find the relationship between the outflow rate and the quantities that appear in the equations of motion.

In the limit of large $\xi \rightarrow \infty$, the dimensionless mass outflow rate $\dot{m}_{\infty}$ is given by the integral

$$
\dot{m}_{\infty}=2 \int_{0}^{\tilde{\omega}_{\infty}} 2 \pi \tilde{\varpi} d \tilde{\varpi}(\alpha u)_{\infty}
$$

where $\tilde{\varpi}=\varpi / R_{P}$ and where the dimensionless outer radius of the outflow $\tilde{\omega}_{\infty}$ can be determined from Equation (28). The leading factor of two arises because the wind flows from both the northern and southern hemispheres of the planet. From Equation (41) we find that $(\alpha u)_{\infty}=H^{1 / 2} u_{1} H_{1}^{-1 / 2}$, where the subscripts " 1 " indicate that the quantities are evaluated at the inner boundary. In the limit $\xi \rightarrow \infty, f \rightarrow \beta, g \rightarrow \beta$, and $H \rightarrow \beta^{2}$. Using the streamline equation, the cylindrical radius $\tilde{\omega}$ is related to the orthogonal coordinate $q$ through the expression $\tilde{\varpi}^{2}=q^{2} / \beta$. After changing variables, the dimensionless outflow rate takes the form

$$
\dot{m}_{\infty}=4 \pi \int_{0}^{q_{X}} u_{1} q d q H_{1}^{-1 / 2}
$$

where $H_{1}$ is a function of $q$ and where $q_{X}^{2}=3 \beta^{1 / 3}$. In general, the starting speed $u_{1}$ will not be the same for all streamlines, i.e., it will be a function of $q$. If we replace $u_{1}$ in the integral by the appropriate mean value $\left\langle u_{1}\right\rangle$, Equation (58) can be evaluated to find

$$
\dot{m}_{\infty}=\frac{4 \pi\left\langle u_{1}\right\rangle(2+\beta)^{2}}{3(1+2 \beta)}\left\{1-\left[1-\frac{9 \beta^{1 / 3}(1+2 \beta)}{(2+\beta)^{3}}\right]^{1 / 2}\right\} .
$$

This expression is valid for $\beta \leqslant 1$. For larger values of $\beta$, the stellar contribution dominates the magnetic field of the planet over its entire surface, and the dimensionless mass outflow rate reduces to the spherically symmetric form $\dot{m}=4 \pi u_{1}$.

The mass outflow rate can also be evaluated at the inner boundary $\xi=1$. The mass outflow rate must be the same in both limits $\xi \rightarrow 1$ and $\xi \rightarrow \infty$, so this calculation provides a consistency check. In this case, the dimensionless outflow rate is given by the expression

$$
\dot{m}_{1}=\int_{S}(\alpha \mathbf{u}) \cdot \hat{r} d S,
$$

where the integral is taken over the entire planetary surface; however, the outflow velocity $\mathbf{u}$ is only nonzero over the fraction of the surface that supports the outflow. Notice also that the flow is not radial, so that the velocity $\mathbf{u}$ points in the $\hat{p}$-direction (rather than the $\hat{r}$-direction). Using the divergence theorem, the integral can be rewritten in the form

$$
\dot{m}_{1}=\int_{V} \nabla \cdot(\alpha \mathbf{u}) d V=\int_{V} \frac{1}{h_{p} h_{q} h_{\phi}} \frac{\partial}{\partial p}\left(\alpha u h_{q} h_{\phi}\right) d V,
$$

where $V$ is the volume of the planet and where $u$ is the only non-vanishing component of the velocity (in the $\hat{p}$-direction). Here, the volume element $d V=h_{p} h_{q} h_{\phi} d p d q d \phi$. The integral with respect to $\phi$ produces a factor of $2 \pi$ because the system is axisymmetric; the integral over $p$ can be evaluated directly and results in a surface term evaluated at the planetary surface $\xi=1$. The remaining expression becomes

$$
\dot{m}_{1}=4 \pi \int_{0}^{q_{X}} u_{1} q d q H_{1}^{-1 / 2},
$$

which has the same form as that in the limit $\xi \rightarrow \infty$ (compare with Equation (58)). Again we must consider flow from both the northern and southern hemispheres of the planet.

Figure 5 shows the dimensionless mass outflow rate $\dot{m}=$ $d m / d t$ plotted as a function of the dimensionless depth $b$ of the gravitational potential well of the planet. The results are shown for a series of values of the field strength parameter $\beta$; the curves correspond to increasing values of $\beta$ from bottom to top in the figure. Notice that the curves end at particular values of $b$ and that these values decrease with increasing $\beta$. For larger values of $b$, only a fraction of the open streamlines allows the flow to pass smoothly through the sonic point. The mass outflow rates are thus diminished. In the discussion below we derive a scaling law (see Equation (64)) that describes how the outflow 


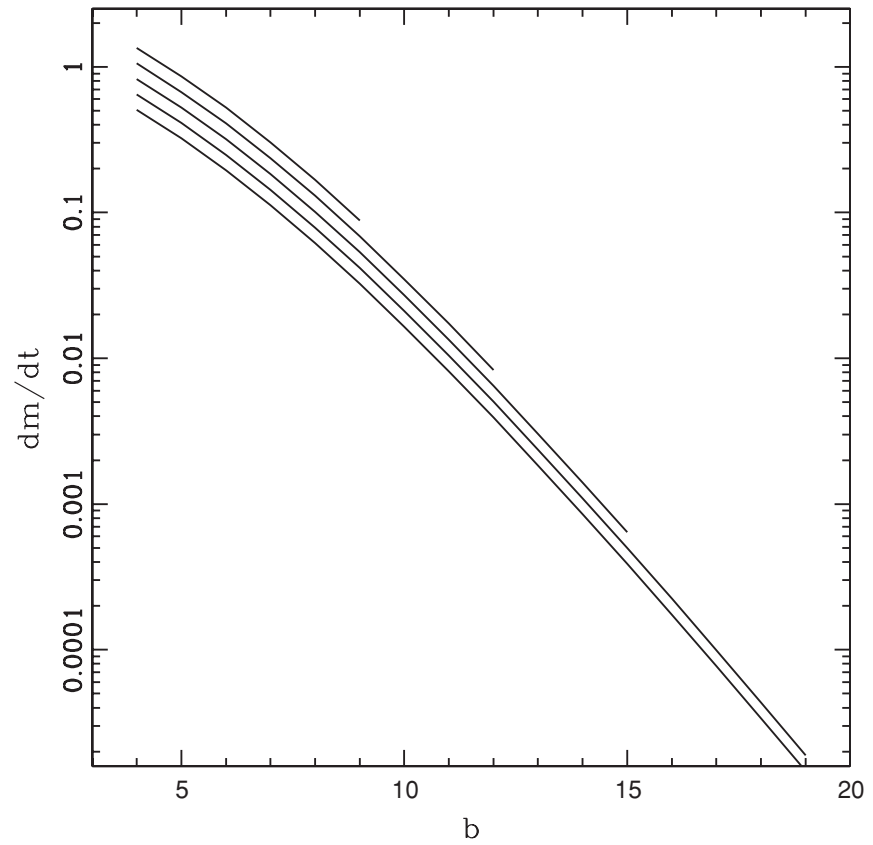

Figure 5. Dimensionless mass outflow rate vs. dimensionless depth $b$ of the gravitational potential well of the planet. The curves show the results for different values of the magnetic field strength parameter $\beta$, from $\beta=0.0005$ (bottom curve) to $\beta=0.008$ (top curve).

rate $\dot{m}$ depends on the variables $(b, \beta)$; over the parameter space represented in Figure 5, this scaling law holds to an accuracy of $\sim 3 \%$.

Figure 6 provides another way to view the dependence of the dimensionless outflow rates on the underlying parameters. In this case, we plot the outflow rate $\dot{m}$ as a function of the field strength ratio $\beta$. Each curve corresponds to a different value of the dimensionless depth $b$ of the gravitational potential well of the planet. The outflow rates increase with $\beta$ until a welldefined maximum is reached; note that the parameters of this extremum are defined by solutions to Equation (52). At this point, further increases in $\beta$ lead to straighter magnetic field lines (and streamlines), especially along the poles of the system, and the flow along those streamlines cannot pass smoothly through the sonic point. As $\beta$ increases, the fraction of the open streamlines that allow for smooth flow decreases, but the fraction of streamlines that are open increases. In addition, the sonic point moves inward, for those streamlines where it exists, and this effect acts to increase the outflow rate $\dot{m}$. These competing effects thus lead to the non-monotonic behavior shown in Figure 6.

The parameter $\beta$ is typically small; for example, $\beta \sim 10^{-3}$ when the field strengths are equal on the stellar and planetary surfaces and $a=10 R_{*} \approx 0.05 \mathrm{AU}$. As a result, it is useful to find simplified results that are correct to leading order in $\beta$. If we expand Equation (59), the leading-order term becomes

$$
\dot{m}=3 \pi\left\langle u_{1}\right\rangle \beta^{1 / 3}+\mathcal{O}\left(\beta^{2 / 3}\right) .
$$

Note that in the case of a spherically symmetric flow, the dimensionless mass-loss rate would have the form $\dot{m}_{\mathrm{sph}}=4 \pi u_{1}$ in these units. The fraction of the total possible outflow rate (with fixed $\left.u_{1}=\left\langle u_{1}\right\rangle\right)$ is thus $F \approx 3 \beta^{1 / 3} / 4$. From the previous section, the fiducial value of the parameter $(\lambda / q)_{0} \sim u_{1} \sim b^{3} \exp [-b]$ (see Equation (53)). We thus except the dimensionless mass

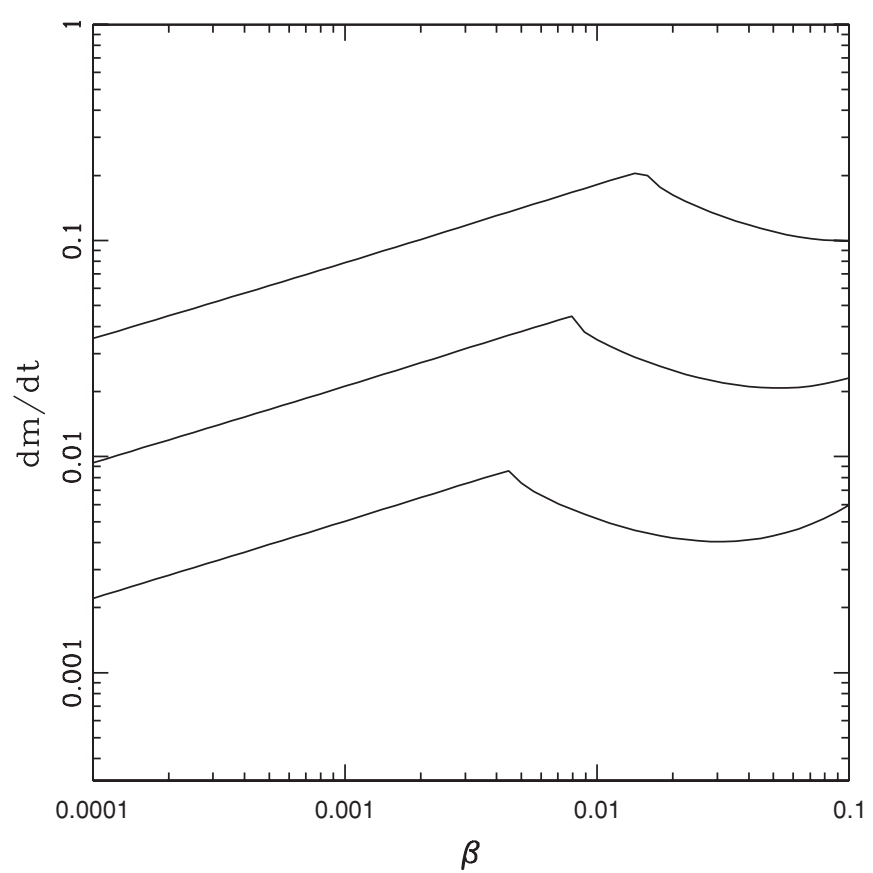

Figure 6. Dimensionless mass outflow rate vs. magnetic field strength parameter $\beta$. The curves show results for different values of the dimensionless depth $b$ of the gravitational potential well of the planet, where $b=12$ (bottom), $b=10$ (middle), and $b=8$ (top). Local maxima occur for the largest values of $\beta$ that allow the flow along all of the open streamlines to pass smoothly through the sonic point (see the text).

outflow rate $\dot{m}$ to obey the scaling relation

$$
\dot{m} \approx A_{m} b^{3} \exp [-b] \beta^{1 / 3},
$$

where $A_{m}$ is a constant of order unity. Fitting to the results presented in Figures 5 and 6 , we find $A_{m} \approx 4.8 \pm 0.13$ (where the quoted uncertainties represent the standard deviation of $A_{m}$ for the parameter space depicted in the figures). This scaling law works well as long as the flow along all of the open field lines can pass through the sonic point. For sufficiently large $\beta$, however, sonic transitions cannot take place in the polar direction, and the dependence of the outflow rate $\dot{m}$ on the parameters $(b, \beta)$ becomes more complicated.

In the limit of large $\beta$, sonic transitions cannot take place along any of the directions. On the other hand, all of the field lines from the planet must match onto stellar field lines. In this case, the values of $\lambda / q$ are given by Equation (56) for all of the streamlines. The integral that defines the dimensionless outflow rate can be evaluated to obtain the result

$$
\dot{m}=6 \pi \beta^{-2 / 3} \exp [-(b+1 / 2)] .
$$

This expression is only valid for $\beta<1$. At larger stellar field strengths, all of the field lines originating on the planet surface are open, and the leading coefficient in Equation (65) becomes $2 \pi(1+2 / \beta)$. In the extreme limit $\beta \gg 1$, the dimensionless outflow rate thus reduces to the expression $\dot{m}=2 \pi \exp [-(b+1 / 2)]$, which is the form expected for flow in the $\hat{z}$-direction from a disk with the radius of the planet.

\subsection{Estimating the Physical Constants}

The previous subsections specify the solutions for the dimensionless fluid fields, including the necessary conditions for passing smoothly through the sonic point and specification of the 
dimensionless mass outflow rate $\dot{m}$. In this section, we complete the solution by estimating values for the physical parameters $\rho_{1}$ and $a_{s}$ that determine the full mass outflow rate, where $\dot{M}=$ $\rho_{1} a_{s} R_{P}^{2} \dot{m}$.

We first provide order of magnitude estimates: the planetary radius is given, with a typical value $R_{P} \approx 10^{10} \mathrm{~cm}$. Since these planets are gas giants, they do not have solid surfaces. As a result, the planetary radii measured by transits are determined by the levels in the atmosphere that are opaque to optical photons, typically at pressures $\sim 1$ mbar (see, e.g., Charbonneau et al. 2007). We also note that the launching radius where $\xi=1$ could lie several scale heights above the planetary surface defined by transit measurements (e.g., at pressures $\sim 1$ nbar). However, the scale height $H \approx R_{P} / b$, where $b \sim 10$, or larger. As a result, $H \ll R_{P}$, and any departures of the launching radius from $R_{P}$ are expected to be small ( $\left.\sim 10 \%\right)$. The gas temperature is expected to approach the benchmark value $T \sim 10^{4} \mathrm{~K}$, so that sound speed $a_{s} \sim 10 \mathrm{~km} \mathrm{~s}^{-1}$. Finally, the density $\rho_{1}$, or equivalently $n_{1}=\rho_{1} / m_{P}$, can be estimated by using the fact that the wind is launched near the $\tau=1$ surface. The optical depth $\tau \sim \sigma_{\mathrm{uv}} n_{1} H$, so the number density $n_{1} \sim 1 /\left(H \sigma_{\mathrm{uv}}\right) \approx$ $b /\left(R_{P} \sigma_{\mathrm{uv}}\right) \sim 10^{9} \mathrm{~cm}^{-3}$. With these values, the scale for the mass outflow rate is $\dot{M}_{\text {scale }}=m_{P} n_{1} a_{S} R_{P}^{2} \sim 10^{11} \mathrm{~g} \mathrm{~s}^{-1}$ (see also MCM). Since the dimensionless factor $\dot{m} \sim 0.01$ (see Figures 5 and 6, and Equation (64)), typical outflow rates are expected to be $\dot{M} \sim 10^{9} \mathrm{~g} \mathrm{~s}^{-1}$.

To obtain a better estimate for the quantities $\left(a_{s}, n_{1}\right)$, we need to consider heating and cooling of the gas (Equations (32), (34), and (35)) including ionization (Equation (36)). Unlike previous outflow studies, the geometry of the flow is determined by the magnetic field structure. For relatively large $\beta$, the magnetic field of the star dominates, and the streamlines become primarily vertical. In the opposite limit of small $\beta$, only the streamlines from the polar regions lead to outflow, and these streamlines are also oriented mostly in the $\hat{z}$-direction (see Figure 2). In either case, the flow is directed in the polar directions, whereas stellar heating arrives from the equatorial direction. Because of this configuration, the optical depth of the incoming radiation is not directly tied to the mass outflow rate (which occurs for spherical flow). In addition, the stellar UV photons penetrate (from the side) into the atmospheric layers where the flow speeds are small. Leaving a full three-dimensional treatment of the heating/cooling problem for the future, we adopt here a simplified approach where flow velocities are neglected. As a further approximation, we assume that the required values of the sound speed $a_{s}$ and density $n_{1}$ are determined at the layer where the optical depth is unity, where $n_{1}=\rho_{1} / m_{P}$ is the total number density at the $\tau=1$ surface.

In this limit, the energy Equation (32) reduces to the condition that heating and cooling are locally in balance, so that $\Lambda=\Gamma$. Similarly, the ionization Equation (36) reduces to the statement that the rate of ionization balances the recombination rate. These two equations can be combined to determine the ionization fraction as a function of temperature. With the definition $X_{+} \equiv n_{+} / n_{0}$, we can write

$$
\begin{aligned}
X_{+}(T) & =\frac{n_{+}}{n_{0}}=\frac{C}{\eta_{\mathrm{abs}}\langle h \nu\rangle \alpha_{R}} \exp \left[-T_{C} / T\right] \\
& \approx\left(2.5 \times 10^{5}\right) T_{4}^{0.9} \exp \left[-11.8348 / T_{4}\right] .
\end{aligned}
$$

With the ionization fraction specified, the heating equation determines the temperature and can be written in the form

$$
\begin{aligned}
\exp \left[-T_{C} / T\right] \frac{X_{+}}{1+X_{+}}=\frac{\eta_{\mathrm{abs}} F_{\mathrm{UV}} \sigma_{\mathrm{uv}}}{C n} \exp [-\tau] \\
=\frac{\eta_{\mathrm{abs}} F_{\mathrm{UV}} \sigma_{\mathrm{uv}}}{C \mathrm{e}} n_{1}^{-1}
\end{aligned}
$$

where $n=n_{0}+n_{+}$is the total number density and $n_{1}=$ $n(\tau=1)$. The left-hand side of Equation (67) is a function of temperature, whereas the right-hand side is a function of position only. The second equality specializes to the layer where $\tau=1$. If we assume that the flow velocities are small, the optical depth is given by

$$
\tau=\frac{n_{1} \sigma_{\mathrm{uv}} R_{p}}{b\left(1+X_{+}\right)}=1,
$$

where $b$ is the dimensionless depth of the gravitational potential well (and note that $b \propto 1 / T$ ). Equations (67) and (68) provide two equations for the two unknowns $n_{1}$ and $T$. With these parameters specified, we can then evaluate the scale $\dot{M}_{\text {scale }}=m_{P} n_{1} a_{s} R_{P}^{2}$ for the mass outflow rate. The outflow rate itself is given by $\dot{M}=\dot{m}\left(m_{P} n_{1} a_{s} R_{P}^{2}\right)$, where the dimensionless outflow rate $\dot{m}$ can be approximated using the scaling law from Equation (64) or the full calculation in that section. Note that in order to determine the value of $b=G M_{P} / a_{s}^{2} R_{P}$, we need the temperature $T \propto a_{s}^{2}$.

Figure 7 shows the number densities $n_{1}$ and corresponding outflow rates $\dot{M}$ as a function of the UV flux $F_{\mathrm{UV}}$ for three choices of planetary mass: $M_{P}=0.5,0.75$, and $1.0 M_{J}$. Keep in mind that the dimensionless outflow rate $\dot{m}$ depends on the magnetic field strength parameter $\beta$ (see Figure 6); these curves are calculated using $\beta=0.001$. In this approximation, the outflow rate increases somewhat more slowly with $F_{\mathrm{UV}}$ than the linear relation of Equation (1). For sufficiently large flux levels (not shown), the temperature approaches its effective maximum value (just above $10^{4} \mathrm{~K}$, see Spitzer 1978); in this regime, the outflow rates would approach a constant value, but the assumptions of this section break down. Notice also that the number density (dashed curves) is a slowly varying function of both the UV flux and the planet mass, and that $n_{1} \sim 10^{9} \mathrm{~cm}^{-3}$ as expected.

\section{OBSERVATIONAL SIGNATURES}

Observations used to infer the presence of planetary outflows show that the transit depth is greater at UV wavelengths than in the optical (Vidal-Madjar et al. 2003, 2004; Désert et al. 2008; Sing et al. 2008; Lecavelier des Etangs et al. 2008; Linsky et al. 2010). As a result, we need to determine the optical depth of the outflow to UV radiation. To start, we define the dimensionless column density $N_{\mathrm{c}}$ according to

$$
N_{\mathrm{c}} \equiv \int_{-\infty}^{\infty} \alpha d s,
$$

where $\alpha$ is the dimensionless density obtained from the flow solution (see Section 4). The column density $N_{\mathrm{c}}$ is thus defined for a given path and the variable $s$ is the dimensionless distance along the path.

Note that Equation (69) determines the total column density, whereas the optical depth depends on the chemical species that absorbs the UV radiation. Most observations to date are carried out for Ly $\alpha$ photons, so that the column density of 


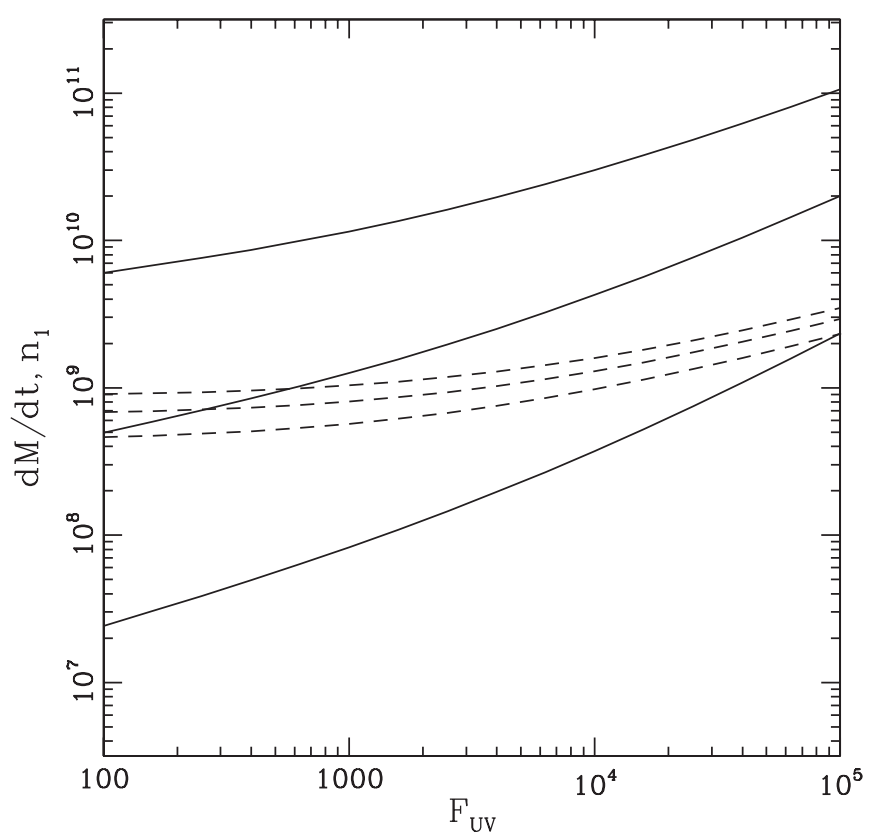

Figure 7. Mass outflow rates as a function of the UV flux from the star. The outflow rate $d M / d t=M$ is given in $\mathrm{g} \mathrm{s}^{-1}$, and the UV flux is given in erg $\mathrm{cm}^{-2} \mathrm{~s}^{-1}$. Solid curves show the outflow rates for three values of the planet mass, from $M_{P}=0.5 M_{J}$ (top curve) to $M_{P}=1.0 M_{J}$ (bottom curve). Dashed curves show the number density $n_{1}\left(\right.$ in $\mathrm{cm}^{-3}$ ) at the $\tau=1$ surface for the same cases. The magnetic field strength parameter $\beta=0.001$ for all cases shown.

interest is that of neutral hydrogen. As a result, ionization modeling is necessary to determine the optical depths and hence the observational signatures. This present treatment assumes isothermal flow. To consistent order of approximation, the neutral column density is reduced from that of Equation (69) by a factor $\mathcal{F}=\left(1+X_{+}\right)^{-1}$ (see Equations (67) and (68)). For the benchmark temperature $T=10^{4} \mathrm{~K}, X_{+} \approx 1.8$, so that the neutral hydrogen column density is reduced to a fraction $\mathcal{F} \approx 0.36$ of the total column density. However, the ionization fraction is a sensitive function of temperature. For $T=8000 \mathrm{~K}$, $X_{+} \approx 0.08$, and the factor $\mathcal{F} \approx 0.93$.

For the sake of definiteness, we evaluate the column density on paths that are parallel to the star-planet direction $(y=0$ in the coordinate system used here; see Figure 1) with a constant vertical height $z$. Each point along the path corresponds to a different streamline, and hence a different value of the constant $\lambda / q$. Figure 8 shows the dimensionless column density $N_{\mathrm{c}}$ through the outflow, as a function of $z$, for several typical cases. The solid curves show the effect of varying the depth of the gravitational potential well of the planet. The three curves correspond to $b=8,10$, and 12, where the magnetic field strength parameter $\beta=0.001$, which corresponds to equal surface fields on the star and planet (for $a=10 R_{*}$ ). The dashed curve shows the effect of doubling the field strength parameter $\beta$ (for $b=10$ ).

The optical depth at UV wavelengths is given by $\tau=$ $\mathcal{F} n_{1} \sigma_{\mathrm{uv}} R_{P} N_{\mathrm{c}}$. For typical cases, the quantity $n_{1} \sigma_{\mathrm{uv}} R_{P} \sim 20$. The base density $n_{1}=\rho_{1} / m_{P}$ is a slowly increasing function of the incident UV flux $F_{\mathrm{UV}}$ (see Figure 7 ), but the correction factor $\mathcal{F}$ due to ionization decreases with temperature and hence decreases with increasing $F_{\mathrm{UV}}$. The range in $z$ for which the flow is optically thick is thus relatively small, ranging from $z / R_{P}=$ 1.35 to 1.77 for the cases shown with $b=8$ to 12 . With these values, the area for which the planet is apparently optically thick

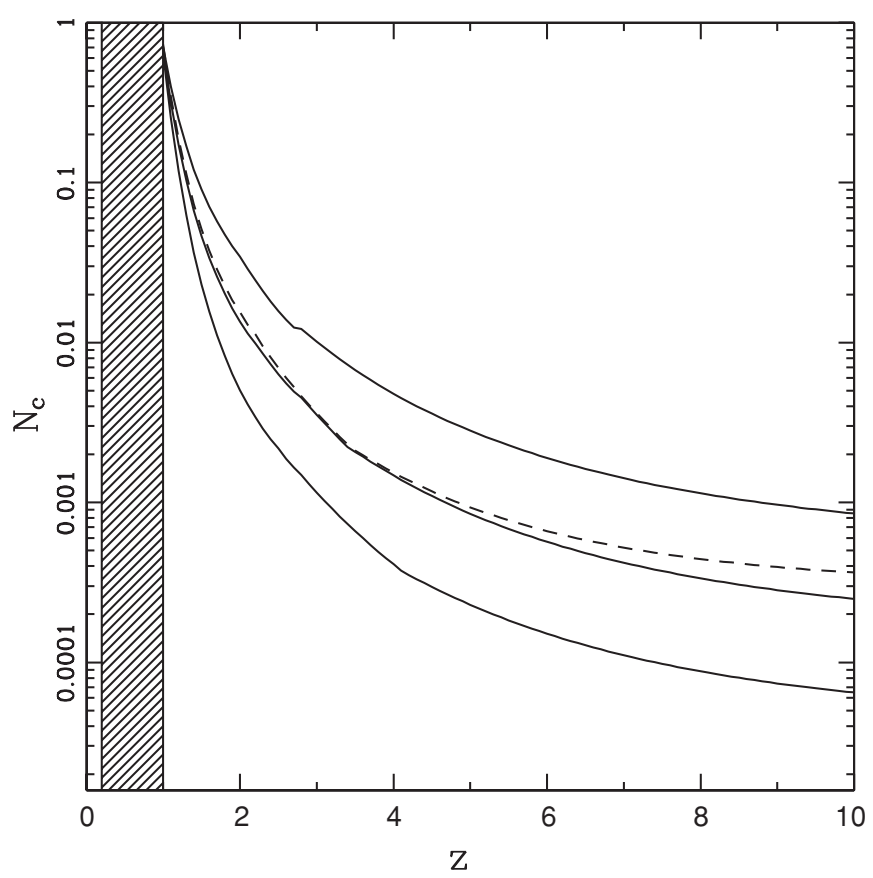

Figure 8. Dimensionless column density through the outflow as a function of vertical coordinate $z$ (in units of the planet radius $R_{P}$ ). Solid curves show the column density for magnetic field parameter $\beta=0.001$ with three values of the dimensionless depth $b$ of the planetary gravitational potential well: $b=8$ (top), 10 (middle), and 12 (bottom). The dashed curve shows the $b=10$ case with larger field strength parameter $\beta=0.002$. Optical depth is obtained from the dimensionless column density through the relation $\tau=\mathcal{F} n_{1} \sigma_{\mathrm{uv}} R_{P} N_{\mathrm{c}} \sim 20 N_{\mathrm{c}}$.

will be 2-3 times larger at UV wavelengths than in the optical bands. The transits for UV observations are thus predicted to be 2-3 times deeper.

The mass outflow rates are expected to vary substantially from planet to planet. In order to illustrate this trend, in Figure 9 we plot estimates for the mass outflow rates for the collection of extrasolar planets that are observed in transit (data from Schneider 2010). The planetary masses, radii, and semimajor axes are observed. In order to estimate the outflow rates, we must also specify the UV flux $F_{\mathrm{UV}}$ and the magnetic field strength parameter $\beta$. With these quantities determined, the results of Section 4 can be used to calculate the mass outflow rate $\dot{M}$. The UV fluxes from main-sequence stars vary with spectral-type and other stellar parameters (e.g., rotation rates) in a complicated manner (see Lecavelier des Etangs 2007; Lammer et al. 2003, and references therein). To obtain the results shown in Figure 9, we estimate the UV flux using a simple scaling law that is intermediate between the scaling laws advocated by the aforementioned authors. To specify the magnetic flux parameter $\beta$, we assume that the stellar and planetary surface fields are comparable, so that to leading order $\beta \propto a^{-3}$. Finally, we take into account the fact that radius of the $\tau=1$ surface (where the outflow is launched and the parameter $b=G M_{P} / a_{s}^{2} R$ is evaluated) lies above the planetary surface at $R_{P}$. Using a simple hydrostatic model for the lower layers of the outflow region, we estimate that $R_{1} \approx 1.2 R_{P}$, and this correction is used here.

With the specifications described above, we obtain the mass outflow rates shown in Figure 9 as a function of planet mass. Several trends are clear: first, for a given planet mass, a wide range of outflow rates are possible. Both the magnetic field parameter $\beta$ and the UV flux $F_{\mathrm{UV}}$ vary with semimajor axis $a$, so that closer planets are predicted to have stronger outflow rates. 


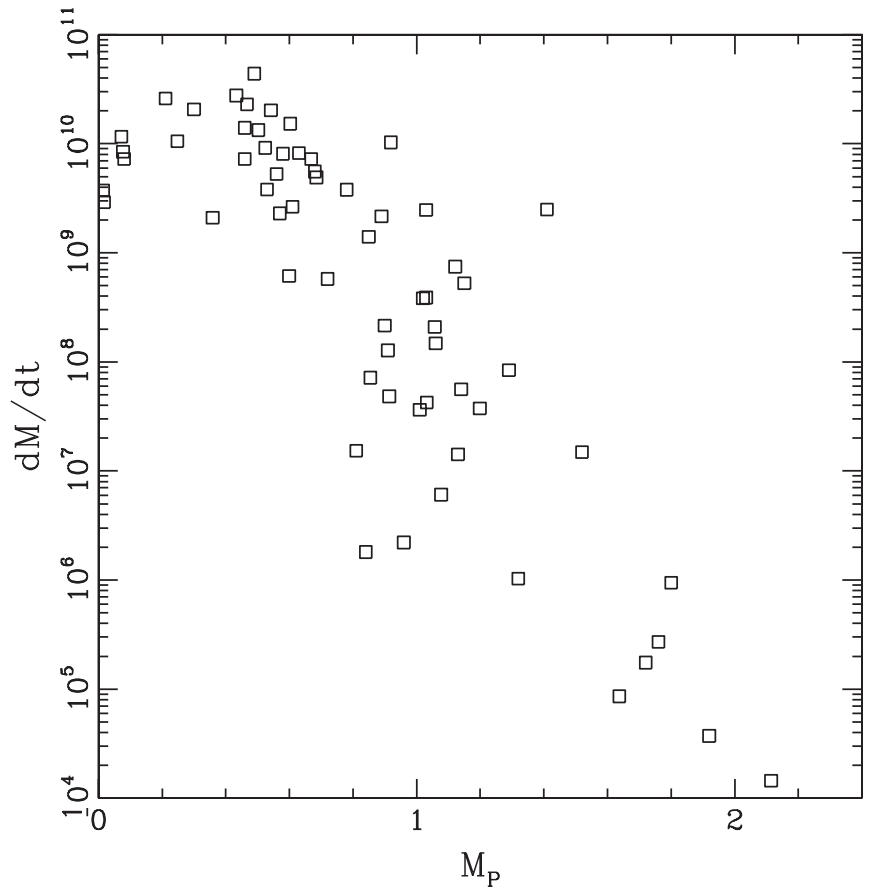

Figure 9. Rough estimates for mass outflow rates for the sample of extrasolar planets observed in transit. Mass outflow rates $d M / d t=\dot{M}$ are given in $\mathrm{g} \mathrm{s}^{-1}$; planetary masses are given in Jovian masses $M_{J}$.

In addition to this dependence on $a$, however, both variables $\left(\beta, F_{\mathrm{UV}}\right)$ are expected to display substantial variation from system to system. Next, we note that the range of outflow rates shown in Figure 9 spans seven orders of magnitude. Those planets with the largest outflow rates should thus have observable transit signatures, whereas those on the other end of the range will show little or no effect. Finally, the outflow rates are predicted to be a steeply decreasing function of planetary mass $M_{P}$. In the isothermal limit, we obtain the nearly exponential decrease shown in Figure 9 (see Equation (64)). If the gas can cool substantially before it passes through the sonic transition, this decrease could be less steep, but the overall trend remains.

Given that two observed planets have signatures of planetary outflows, it is useful to see how they compare with the results shown in Figure 9. The planet HD209458b (Vidal-Madjar et al. 2003) has mass $m_{P}=0.685 m_{J}$, and the figure shows that the expected outflow rates are $\dot{M} \sim 10^{10} \mathrm{~g} \mathrm{~s}^{-1}$. Even with the reduction due to magnetic effects, such estimated outflow rates are large enough to account for the observations of HD209458b (although these values are near the low end of the inferred range). However, for the planet HD189733b (Lecavelier des Etangs et al. 2010), the mass is larger, $m_{P}=1.15 m_{J}$, and the predicted outflow rate from this theory is much lower. The values shown in Figure 9 correspond to $\dot{M} \sim 10^{8} \mathrm{~g} \mathrm{~s}^{-1}$ with significant scatter. The observational papers for HD189733b advocate a large UV flux (up to 40 times the solar value), which increases the theoretical outflow rate to $\dot{M} \sim 10^{9} \mathrm{~g} \mathrm{~s}^{-1}$ for our choice of field strength parameter $\beta=10^{-3}$. To account for the inferred outflow rate of $\dot{M} \sim 10^{10} \mathrm{~g} \mathrm{~s}^{-1}$, one could invoke a larger value of $\beta$ for this system. Nonetheless, some tension remains between the inferred outflow rate and that expected theoretically from magnetically controlled models. More observations, of this system and others with a range of masses, are needed to sort out this comparison.

\section{CONCLUSION}

\subsection{Summary of Results}

This paper has begun a theoretical study of outflows from the surfaces of hot Jupiters in the regime where the flow is controlled by magnetic fields. In this case, the magnetic field structure determines the flow geometry (whereas the field configurations are determined by independent dynamo processes within the planet and by the background contribution from the star). With the magnetic field structure specified, the dimensionless version of the outflow problem can be solved semi-analytically; this paper carries out this calculation in the isothermal limit, including the requirement that the flow must pass smoothly through the sonic transition. The determination of the physical constants represents the final piece of the calculation. The specific results of this paper can be summarized as follows.

1. This paper considers a reduced description of the magnetic field structure that includes the dipole field of the planet and a constant background contribution. The reduced field structure near the planet is modeled in Section 3 and provides a description of the flow geometry in the region where the outflow is launched (see Figure 2). In this section, we construct the corresponding coordinate system and differential operators for this flow geometry. These results are used here to study the launch of outflows for hot Jupiters, but can also be used in a variety of other applications.

2. A fraction $F_{\mathrm{AP}}$ of the planetary surface supports magnetic field lines that are open with respect to the planet. This area fraction provides a constraint on the mass flow that can fully leave the planet. The remaining fraction, $1-F_{\mathrm{AP}}$, defines the region where material can leave the planetary surface but is nonetheless confined to the immediate vicinity of the planet; this material produces an exosphere surrounding the planet. In the magnetically controlled regime, the outflow rate is thus lower than estimates obtained by assuming spherical symmetry. The fraction $F_{\mathrm{AP}}$ is calculated analytically for the reduced field configuration near the planet (Section 3 ).

3. Along each streamline, the dimensionless energy $\varepsilon$ and the flow momentum parameter $\lambda$ are constant, but vary across streamlines. These parameters are specified by requiring that the flow pass smoothly through the sonic point and by the boundary conditions at the planetary surface. The resulting specification of parameters can be found analytically (see Equations (50) and (51), and Figure 3 ). The outflow rate is determined by integrating over the surface area of the planet where the outflow is active, where the angle of the flow direction (which is not radial) must be included (see Equations (57)-(64)). The resulting mass outflow rates are well-defined functions of the depth $b$ of the planetary potential well and the magnetic field strength parameter $\beta$ (see Figures 5 and 6). Over the regime of parameter space where flow along all of the open field lines can pass smoothly through the sonic point, the scaling law of Equation (64) provides an accurate determination of the dimensionless outflow rate $\dot{m}$.

4. This flow geometry is significantly different from previous cases that assume spherical symmetry. For magnetically controlled outflows, only a fraction of the field lines (and hence streamlines) allow outflow. In addition, passage through the sonic point depends sensitively on the divergence operator (at the transition) which depends on the configuration of the streamlines. As the magnetic field strength of the background (from the star) increases relative 
to that of the planet, more of the surface has open field lines, but not all of the streamlines allow for smooth sonic transitions. Another key difference is that spherical flows are often taken to be "self-limiting," where the outflow rate, in part, determines the optical depth to the incoming photons. In this geometry, most of the heating photons impinge upon the system from the equatorial directions, whereas most of the outflow is directed along the poles of the planet. This flow geometry also changes the effect of tidal forces exerted on the planet by the star: because mass loss occurs along the polar directions, the tidal forces act to inhibit, rather than enhance, the mass outflow rates (see also TAL; compare with MCM).

\subsection{Discussion}

Since this problem contains a number of physical parameters, it is useful to summarize them here and discuss which ones are the most important. The structure of the magnetic field requires the specification of three quantities: the surface field strength $B_{P}$, the effective planetary radius $R_{P}$, and the ratio $\beta$ of the background field strength to the surface field strength. For the cases under consideration, however, the magnetic field is assumed to be strong enough to guide the flow, so that only the field geometry plays a role. In this simplified treatment, the magnetic field geometry is characterized by the single parameter $\beta$ (see Equation (11)). For purposes of launching the wind from the planet, the main contribution of the stellar field is to provide a nearly constant background field, taken here to lie in the vertical direction. Since the planetary field is much stronger in the region within the sonic surface, this assumption is valid for determining the launch of the wind. However, the stellar field direction will not necessarily line up with the pole of the planet, so that more general geometries should be considered in the future. In addition, the stellar field configuration will affect the manner in which the outflow propagates after its launch-beyond the sonic surface - and this problem should also be addressed in future work.

With the flow geometry set by the magnetic field structure, the outflow problem determines the fluid variables as a function of the (single) coordinate $p$ which follows the field lines (Section 4). These quantities include the density $\rho(p)$, flow speed $v(p)$, temperature $T(p)$, and ionization fraction $X_{+}(p)=$ $n_{+} / n_{0}$, and depend on the planet mass $M_{P}$ and radius $R_{P}$, and the stellar heating flux $F_{\mathrm{UV}}$. Under the assumption of isothermal flow, the problem is reduced further to two dimensionless fluid variables $\alpha=\rho / \rho_{1}$ and $u=v / a_{s}$, along with the dimensionless depth of the planetary gravitational well $b=G M_{P} /\left(a_{s}^{2} R_{P}\right)$. The dimensionless problem thus has only two parameters $(b, \beta)$, and they determine the dimensionless outflow rate (see Equations (57)-(65) and Figures 5 and 6).

The determination of physical quantities requires specification of the density scale $\rho_{1}$, the sound speed $a_{s}$, and the planet radius $R_{P}$. The UV flux $F_{\mathrm{UV}}$ from the star determines, in part, the sound speed $a_{s}$ and the density $\rho_{1}$ at the base of the flow. Over the expected parameter space, the sound speed $a_{s}$ and density scale $n_{1}$ vary by only factors of $3-10$. However, the outflow rate decreases exponentially with the depth $b$ of the potential well and shows complicated dependence on the magnetic field strength parameter $\beta$ (see Figures 5, 6, and 7). Note that this exponential sensitivity to the depth of the potential well is a common feature in outflow problems where the sound speed is less than the escape speed (compare with the case of outflows from circumstellar disks driven by external FUV radiation; Adams et al. 2004). Since the dimensionless outflow rate displays exponential dependence on $b$, where $b=G M_{P} / R a_{s}^{2}$, the exact value of the temperature (or equivalently the sound speed) can be important. In addition, the radius $R$ in this expression corresponds to the radius $R_{1}$ where the outflow is launched, i.e., the $\tau=1$ surface. Although the radius $R_{1}$ differs from the planet radius $R_{P}$ by only $\sim 10 \%$, this difference can be significant for the regime of large $b$ where the outflow rates are exponentially suppressed.

\subsection{Future Work}

This paper represents only the first step toward understanding outflows from hot Jupiters in the regime where magnetic fields dominate the flow geometry. This work should be carried forward in a number of directions.

One approximation used here is the assumption of isothermal flow. For the next stage of development, an analogous calculation can be carried out using a more general, polytropic equation of state. However, a full treatment of the heating and cooling should be undertaken. This calculation requires one to solve the energy equation (32) and the ionization equation (36), where the heating from the central star is determined through a threedimensional radiative transfer calculation. The chemistry of the outflow should also be included, both to get a better description of the heating and cooling processes and to determine observational signatures (e.g., García Muñoz 2007). Note that one of the intrinsic complications that arises in this problem is that the flow configuration (determined by magnetic field lines) does not have the same geometry as the heating and cooling processes.

This paper considers a dipole magnetic field on the planet and a constant background field from the star; in addition, this work specializes to the case where the stellar contribution to the magnetic field near the planet is purely vertical. A wide range of magnetic field configurations are possible in star/planet systems and these possibilities should be explored further. One particular issue arises with sufficiently strong stellar fields, which provide a background field for purposes of launching the wind from the planet. If the stellar field is sufficiently strong and straight, the flow cannot pass smoothly through a sonic transition. In this case, the sonic point is effectively removed to spatial infinity and the outflow rates are suppressed (e.g., see Equation (65)). For the geometry considered here this suppression arises when the field strength parameter $\beta \gtrsim 0.01$ (which requires the stellar surface field to be $\sim 10$ times that of the planet for $a=0.05 \mathrm{AU}$, or equal to that of the planet for $a=0.023 \mathrm{AU})$. A more detailed study of this regime should be undertaken, including more complicated configurations for the background (stellar) field. Another related effect is that the interaction between the planetary magnetic field and the stellar magnetosphere, including the flow of material considered here, can lead to an enhancement in stellar activity (e.g., Cuntz et al. 2000; Cohen et al. 2009) and orbital evolution (Chang et al. 2010). This paper also focuses on the case where the stellar field is vertical, which would arise, e.g., from a dipole field from the star that rotates with the same angular velocity as the planetary orbit. Future work should relax this assumption. In particular, the field lines could trail the planet, wrap up, and lead to further complications.

For sufficiently strong magnetic fields and low outflow rates, the ram pressure of the flow is not strong enough to affect the underlying field structure. For weaker fields and/or higher outflow rates, however, the back-reaction of the flow on the magnetic field should be taken into account. An understanding of this physics can be attained through the solution to the 
Grad-Shafranov equation (e.g., Shafranov 1966), which selfconsistently determines the distribution of streamlines (see also Cai et al. 2008). Even for the strong field limit, considered here, this back-reaction plays a role near the X-point. On a related note, the closed field lines can also be loaded with mass and the planet will thus develop a quasi-static exosphere (see TAL); this exosphere can enhance the observational signature, by increasing the transit depth at UV wavelengths, and should thus be considered further.

Finally, we note that some of the planets observed in transit have orbits with nonzero eccentricity. The canonical example is the planet HD17156b (Barbieri et al. 2007), which has an orbital eccentricity of $e \approx 0.67$; in this system, the stellar flux varies by a factor of $\sim 25$ over the course of the planetary orbit. The planetary outflow in this system, and others with similar architecture, will thus be time dependent. The resulting timedependent outflow rates will be sensitive functions of the heating and cooling mechanisms, as well as the (complicated and timedependent) magnetic field configurations. The study of such systems will provide sensitive tests of the outflow mechanism.

This paper benefited from discussions with many colleagues, especially Phil Arras, Mike Cai, Daniele Galli, Zhi-Yun Li, Greg Laughlin, Susana Lizano, Nathan Schwadron, and Frank Shu. An anonymous referee provided many useful comments and suggestions. This work was supported at the University of Michigan through the Michigan Center for Theoretical Physics. Portions of this work were carried out at the Kavli Institute for Theoretical Physics at the University of California, Santa Barbara (supported by the National Science Foundation under grant no. PHY05-51164). F.C.A. is supported by NASA through the Origins of Solar Systems Program (grant NNX07AP17G) and by NSF through the Division of Applied Mathematics (grant DMS-0806756).

\section{APPENDIX}

\section{EQUIVALENT SPHERICAL PROBLEM}

One complication inherent in the study of magnetically controlled outflows is that the problem is three dimensional. Even the reduced problem considered here, with a constant stellar field, requires two dimensions. In order to gain further insight into the problem, this appendix develops an equivalent spherically symmetric version of the wind problem.

The geometry of the flow pattern is specified by the divergence operator. The quantity that appears in the conditions that match solutions at the sonic point can be written as

$$
G_{\mathrm{op}} \equiv \mathcal{G}\left(\frac{\partial \xi}{\partial p}\right)^{-1},
$$

where the geometrical factor $\mathcal{G}$ is given by Equation (46) and $\partial \xi / \partial p$ is given by Equation (47). In the limit of large $\xi \gg 1$, one can show that $G_{\mathrm{op}} \sim 12 /\left(\beta \xi^{4}\right)$. In the opposite limit where $\xi \sim 1$, near the planetary surface, $G_{\mathrm{op}} \sim 3 / \xi$. This latter form is consistent with the result for a dipole field configuration near the poles of the system (recall that the outflow is concentrated near the poles). These two limiting forms can be connected through intermediate values by adopting the form

$$
G_{\mathrm{op}}=\frac{3}{\xi\left(1+\beta \xi^{3} / 4\right)} \text {. }
$$

The integrated form of the dimensionless continuity equation then becomes

$$
\alpha u \frac{\xi^{3}}{1+\beta \xi^{3} / 4}=\lambda,
$$

where $\lambda$ is a (single) constant for the equivalent spherical problem. The differential form of the continuity equation is obtained by taking the derivative of Equation (A3). Near the planet, we thus obtain $d\left(\alpha u \xi^{3}\right) / d \xi=0$, the form appropriate for a dipole divergence. Far from the planet, the continuity equation reduces to the form $d(\alpha u) / d \xi=0$, which is the form applicable to flow along a single (Cartesian) direction. The force equation remains the same (see Equation (42)).

With this form for the divergence, the condition for flow passing smoothly through the sonic point takes the form

$$
b=\frac{3 \xi}{1+\beta \xi^{3} / 4},
$$

which thus specifies the radius $\xi_{s}$ of the sonic transition (compare with Equation (52)). In the limit $\beta \rightarrow 0$, this matching condition becomes $b=3 \xi$, as expected for a dipole. This expression (A4) has no real solutions for sufficiently large $b$ and/or large $\beta$. The condition required for solutions to exist, and hence for the flow to pass through the sonic point, can be written in the form

$$
\beta b^{3}<16
$$

For cases where sonic transitions are possible, the required value of the constant $\lambda$ is thus given implicitly by the relation

$$
\frac{1}{2} \lambda^{2}(1+\beta / 4)^{2}-\ln \lambda=\frac{1}{2}+\ln \left(\beta / 4+\xi_{s}^{-3}\right)+b\left(1-1 / \xi_{s}\right),
$$

where $\xi_{s}$ is given by the solution to cubic Equation (A4). For consistency, the area subtended by the outflow must be proportional to the function $\xi^{3} /\left(1+\beta \xi^{3} / 4\right)$ that appears in the continuity Equation (A3). This quantity starts near unity at the planet surface, grows like $\xi^{3}$ near the planet, and then approaches a constant value in the limit $\xi \rightarrow \infty$. This behavior is thus analogous to that of the more physical problem considered in the text.

\section{REFERENCES}

Adams, F. C., Hollenbach, D., Laughlin, G., \& Gorti, U. 2004, ApJ, 611, 360 Baraffe, I., Alibert, Y., Chabrier, G., \& Benz, W. 2006, A\&A, 450, 1221

Baraffe, I., Selsis, F., Chabrier, G., Barman, T. S., Allard, F., Hauschildt, P. H., \& Lammer, H. 2004, A\&A, 419, L13

Barbieri, M., et al. 2007, A\&A, 476, 13

Batygin, K., \& Stevenson, D. J. 2010, ApJ, 714, L238

Black, J. H. 1981, MNRAS, 197, 553

Cai, M. J., Shang, H., Lin, H.-H., \& Shu, F. H. 2008, ApJ, 672, 489

Chang, S.-H., Gu, P.-G., \& Bodenheimer, P. H. 2010, ApJ, 708, 1692

Charbonneau, D., Brown, T. M., Burrows, A., \& Laughlin, G. 2007, in Protostars and Planets V, ed. B. Reipurth, D. Jewitt, \& K. Keil (Tuscon, AZ: Univ. Arizona Press), 701

Christensen, U. R., Holzwarth, V., \& Reiners, A. 2009, Nature, 457, 167

Cohen, O., Drake, J. J., Kashyap, V. L., Saar, S. H., Sokolov, I. V., Manchester, W. B., Hansen, K. C., \& Gombosi, T. I. 2009, ApJ, 704, 85

Cuntz, M., Saar, S. H., \& Musielak, Z. E. 2000, ApJ, 533, L151

Désert, J.-M., Vidal-Madjar, A., Lecavelier des Etangs, A., Sing, D., Ehrenreich, D., Hébrard, G., \& Ferlet, R. 2008, A\&A, 492, 585

Fabrycky, D. A., \& Winn, J. N. 2009, ApJ, 696, 1230

García Muñoz, A. 2007, Planet. Space Sci., 55, 1426

Hubbard, W. B., Hattori, M. F., Burrows, A., Hubeny, I., \& Sudarsky, D. 2007, Icarus, 187, 358

Lai, D., Helling, Ch., \& van den Heuvel, E. P. J. 2010, ApJ, 721, 923

Lammer, H., Selsis, F., Ribas, I., Guinan, E. F., Bauer, S. J., \& Weiss, W. W. 2003, ApJ, 598, 121 
Langton, J., \& Laughlin, G. 2008, ApJ, 674, 1106

Lecavelier des Etangs, A. 2007, A\&A, 461, 1185

Lecavelier des Etangs, A., Vidal-Madjar, A., Désert, J.-M., \& Sing, D. 2008, A\&A, 485, 865

Lecavelier des Etangs, A., et al. 2010, A\&A, 514, 72

Lin, D. N. C., Bodenheimer, P., \& Richardson, D. C. 1996, Nature, 6575, 606

Linsky, J. L., Yang, H., France, K., Froning, C. S., Green, J. C., Stocke, J. T., \& Osterman, S. N. 2010, ApJ, 717, 1291

Lissauer, J. J., \& Stevenson, D. J. 2007, in Protostars and Planets V, ed. B. Reipurth, D. Jewitt, \& K. Keil (Tuscon, AZ: Univ. Arizona Press), 591

Mestel, L. 1968, MNRAS, 138, 359

Mestel, L., \& Spruit, H. C. 1987, MNRAS, 226, 57

Murray-Clay, R. A., Chiang, E. I., \& Murray, N. 2009, ApJ, 693, 23 (MCM)

Papaloizou, J. C. B., \& Terquem, C. 2006, Rep. Prog. Phys., 69, 119

Parker, E. N. 1958, ApJ, 128, 664

Parker, E. N. 1965, Space Sci. Rev., 4, 666

Radoski, H. R. 1967, J. Geophys. Res., 72, 418

Schneider, J. 2010, The Extrasolar Planet Encyclopedia, http://exoplanet.eu

Shafranov, V. D. 1966, Rev. Plasma Phys., 2, 103
Shu, F. H. 1992, Gas Dynamics (Mill Valley, CA: Univ. Science Books)

Shu, F. H., Najita, J., Ostriker, E., Wilkin, F., Ruden, S., \& Lizano, S. 1994, ApJ, 429,781

Sing, D. K., Vidal-Madjar, A., Désert, J.-M., Lecavelier des Etangs, A., \& Ballester, G. 2008, ApJ, 686, 658

Spitzer, L. 1978, Physics Processes in the Interstellar Medium (New York: Wiley)

Stevenson, D. J. 2003, Earth Planet. Sci. Lett., 208, 1

Stone, J. M., \& Proga, D. 2009, ApJ, 694, 205

Storey, P. J., \& Hummer, D. G. 1995, MNRAS, 272, 41

Sturrock, P. A. 1994, Plasma Physics (Cambridge: Cambridge Univ. Press)

Trammell, G. B., Arras, P., \& Li, Z.-Y. 2011, ApJ, 728, 152 (TAL)

Vidal-Madjar, A., Lecavelier des Etangs, A., Désert, J.-M., Ballester, G. E., Ferlet, R., Hébrard, G., \& Mayor, M. 2003, Nature, 422, 143

Vidal-Madjar, A., et al. 2004, ApJ, 604, 69

Watson, A., Donahue, T., \& Walker, J. 1981, Icarus, 48, 150

Weinreich, G. 1998, Geometrical Vectors (Chicago, IL: Univ. Chicago Press)

Woods, T. N., Rottman, G. J., Bailey, S. M., Solomon, S. C., \& Worden, J. R. 1998, Sol. Phys., 177, 133

Yelle, R. V. 2004, Icarus, 170, 167 